\title{
NORMALITY OF ORBIT CLOSURES IN THE ENHANCED NILPOTENT CONE
}

\author{
PRAMOD N. ACHAR, ANTHONY \\ HENDERSON, AND BENJAMIN F. JONES
}

\begin{abstract}
We continue the study of the closures of GL( $V)$-orbits in the enhanced nilpotent cone $V \times \mathcal{N}$ begun by the first two authors. We prove that each closure is an invariant-theoretic quotient of a suitably defined enhanced quiver variety. We conjecture, and prove in special cases, that these enhanced quiver varieties are normal complete intersections, implying that the enhanced nilpotent orbit closures are also normal.
\end{abstract}

\section{$\S 1$. Introduction}

The geometry of nilpotent orbits in complex semisimple Lie algebras is a topic of central importance in numerous branches of representation theory. A fundamental question on this topic is whether the closures of nilpotent orbits are normal varieties. This question was answered in the affirmative for nilpotent orbits in type $A$ by Kraft and Procesi [KP1] in 1979. In other types, the answer turns out to be "not always": an explicit determination of the nilpotent orbits with normal closures was carried out in types $B$ and $C$ by Kraft and Procesi [KP2] and in types $G_{2}, F_{4}$, and $E_{6}$ by Kraft [Kr], Broer [B], and Sommers [S1], respectively. The case of type $D$ was partially resolved by Kraft and Procesi [KP2] and completed by Sommers [S2]. A complete answer is not yet known in types $E_{7}$ and $E_{8}$.

This paper is concerned with the variety $V \times \mathcal{N}$, where $V$ is a finitedimensional complex vector space and $\mathcal{N}$ is the variety of nilpotent elements in $\operatorname{End}(V)$. This variety, known as the enhanced nilpotent cone, was studied

Received April 22, 2010. Revised November 6, 2010. Accepted December 16, 2010.

2010 Mathematics Subject Classification. Primary 17B08; Secondary 14L30.

Achar's research was partially supported by Louisiana Board of Regents grant NSF(2008)-LINK-35 and by National Security Agency grant H98230-09-1-0024. Henderson's research was partially supported by Australian Research Council grant DP0985184. Jones's research was partially supported by National Science Foundation grant DMS0738586 . 
by the first two authors in $[\mathrm{AH}]$. It is closely related to Kato's exotic nilpotent cone (see [Ka1], [Ka2]) and to the work of Travkin [T] together with Finkelberg and Ginzburg [FGT] on mirabolic character sheaves. The geometry of $\mathrm{GL}(V)$-orbits on $V \times \mathcal{N}$ resembles that of ordinary type- $A$ nilpotent orbits in some ways (e.g., the only equivariant local systems are trivial) but is reminiscent of types $B$ and $C$ in others (e.g., the orbits are parameterized by bipartitions, and the local intersection cohomology of orbit closures is described by type- $B / C$ combinatorics (see $[\mathrm{AH}])$ ). The upshot of this paper is that, regarding the normality of orbit closures, the enhanced nilpotent cone is analogous to the type- $A$ nilpotent cone. That is, our results contribute to proving the following generalization of [KP1, Theorem 0.1].

Conjecture 1.1. The closure of each $\mathrm{GL}(V)$-orbit in $V \times \mathcal{N}$ is normal.

In this paper, we prove a series of implications, summarized in Figure 1, that reduces Conjecture 1.1 to a combinatorial statement, Conjecture 6.5. The combinatorics is more complicated than in the unenhanced case studied in [KP1], and at present we can prove Conjecture 6.5 only for a restricted class of enhanced nilpotent orbits, namely, those satisfying the conditions of Proposition 4.10 and those satisfying the conditions of Theorem 6.9. We have also verified it by computer for orbits in low dimensions. The following cases of Conjecture 1.1 are thereby proved. (The notation will be explained in Section 2.)

THEOREM 1.2. The enhanced nilpotent orbit closure $\overline{\mathcal{O}_{\mu ; \nu}}$ is normal in the following cases:

(1) $|\mu+\nu| \leq 6$;

(2) $\mu_{1}^{\mathbf{t}} \leq \nu_{\nu_{1}}^{\mathbf{t}}$ (i.e., every column of the diagram of $\nu$ is at least as long as every column of $\mu$ );

(3) $\mu_{\mu_{1}}^{\mathbf{t}}>\nu_{1}^{\mathbf{t}}$ (i.e., every column of $\mu$ is longer than every column of $\nu$ ).

The main tool in our argument is a new class of spaces called enhanced quiver varieties. These varieties, whose definition (see Section 4) is inspired by the methods of Kraft and Procesi [KP1], seem to be interesting in their own right. In Proposition 4.12, we exhibit the closure of a GL( $V)$-orbit in $V \times \mathcal{N}$ as an invariant-theoretic quotient of an enhanced quiver variety. So, as in [KP1], proving the normality of the enhanced quiver varieties would suffice to prove Conjecture 1.1. 


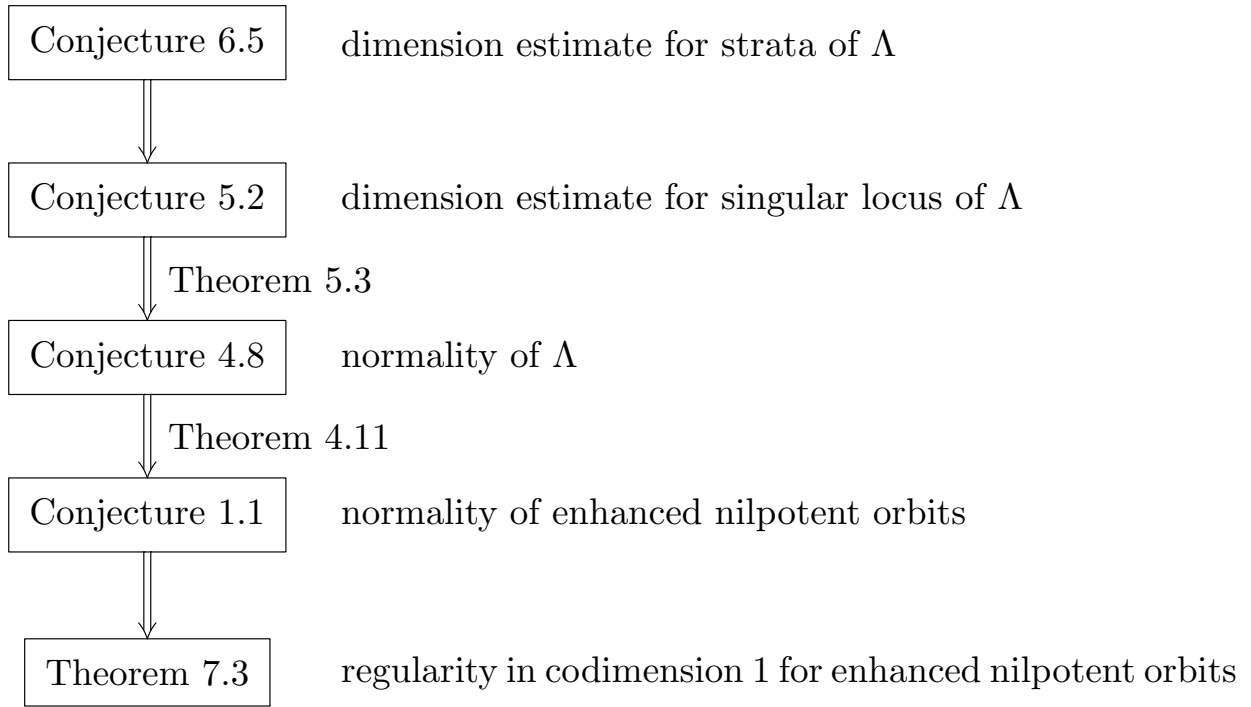

Figure 1

EXAMPLE 1.3. Here is one example to give the flavor of the general definition. Suppose that $\operatorname{dim} V=4$. The closure of the subregular orbit in $\mathcal{N}$ is

$$
\left\{x \in \mathcal{N} \mid x^{3}=0\right\},
$$

which in [KP1] is described as an invariant-theoretic quotient of the following quiver variety: the variety of quadruples $\left(A_{1}, B_{1}, A_{2}, B_{2}\right)$ of linear maps

$$
\mathbb{C}^{1} \underset{B_{1}}{\stackrel{A_{1}}{\rightleftarrows}} \mathbb{C}^{2} \underset{B_{2}}{\stackrel{A_{2}}{\rightleftarrows}} V
$$

satisfying the equations $B_{1} A_{1}=0$ and $B_{2} A_{2}=A_{1} B_{1}$. In the enhanced setting, one of the orbit closures in $V \times \mathcal{N}$ is

$$
\left\{(v, x) \in V \times \mathcal{N} \mid x^{3}=0, x^{2} v=0\right\} .
$$

We will describe this as an invariant-theoretic quotient of the following enhanced quiver variety: the variety of sextuples $\left(u, v, A_{1}, B_{1}, A_{2}, B_{2}\right)$ where $\left(A_{1}, B_{1}, A_{2}, B_{2}\right)$ is as above, $u \in \mathbb{C}^{1}, v \in V$, and $A_{1} u=B_{2} v$. 
We begin in Section 2 by fixing notation and conventions for partitions and related combinatorial objects and by recalling relevant facts about enhanced nilpotent orbits and related varieties. Section 3 is devoted to the proof of a preparatory result on quotients of the space of enhanced nilpotent pairs. Enhanced quiver varieties are introduced in Section 4, which also contains the proof that their quotients are isomorphic to the enhanced nilpotent orbit closures. The next two sections carry out further study of the geometry of enhanced quiver varieties and conclude with a proof of their normality in certain cases: the aforementioned combinatorial conjecture is stated and discussed in Section 6. Finally, in Section 7, which is somewhat independent of the rest of the paper, we prove that all enhanced nilpotent orbit closures are regular in codimension 1. This is, of course, a necessary condition for Conjecture 1.1 to hold, and it is not immediately obvious, because enhanced nilpotent orbits can have orbits of codimension 1 in their boundary.

The results of Section 7 hold over an arbitrary algebraically closed field, which raises the possibility that Conjecture 1.1 may also be true in positive characteristic. The method of proof suggested in this paper follows [KP1] in assuming that the characteristic is zero, but it is possible that it could be adapted to positive characteristic with the techniques used by Donkin [D] in the unenhanced case.

\section{$\S 2 . \quad$ Partitions and nilpotent matrices}

In this section, we fix notation related to the combinatorics of partitions and bipartitions, and we review relevant results on nilpotent orbits, nilpotent pairs, and enhanced versions thereof. These results hold over any field, but we use $\mathbb{C}$ for the sake of subsequent sections.

\subsection{Compositions, partitions, bipartitions}

A composition is a sequence $\lambda=\left(\lambda_{1}, \lambda_{2}, \ldots\right)$ of nonnegative integers with finitely many nonzero terms. The size of a composition, denoted $|\lambda|$, is the sum of its terms. The infinite tail of zeros will typically be omitted when writing a composition.

A partition is a composition $\left(\lambda_{1}, \lambda_{2}, \ldots\right)$ with $\lambda_{1} \geq \lambda_{2} \geq \cdots$. The length $\ell(\lambda)$ of a partition $\lambda$ is the number of nonzero terms. Partitions are often written with exponents indicating multiplicities; for instance, we may write $3^{2} 1^{3}$ rather than $(3,3,1,1,1)$. Let

$$
\mathcal{P}_{n}=\{\text { partitions of size } n\} \text {. }
$$


A bipartition of size $n$ is simply an ordered pair $(\mu ; \nu)$ of partitions with $|\mu|+|\nu|=n$. We put

$$
\mathcal{Q}_{n}=\{\text { bipartitions of size } n\}
$$

Given a bipartition $(\mu ; \nu)$, we can form a partition in two ways: the sum $\mu+\nu$ (obtained by termwise addition of sequences) and the union $\mu \cup \nu$ (obtained by arranging the nonzero terms of $\mu$ and $\nu$ in decreasing order). The transpose $\lambda^{\mathbf{t}}$ of a partition $\lambda$ is given by $\left(\lambda^{\mathbf{t}}\right)_{i}=\#\left\{j \mid \lambda_{j} \geq i\right\}$. Note that $(\mu+\nu)^{\mathbf{t}}=\mu^{\mathbf{t}} \cup \nu^{\mathbf{t}}$.

A convenient way to visualize partitions and bipartitions is via diagrams of boxes. For a partition $\lambda$ we use the usual left-justified Young diagram where the parts of $\lambda$ give the number of boxes in each row and where the parts of $\lambda^{\mathbf{t}}$ give the number of boxes in each column. For a bipartition $(\mu ; \nu)$, following $[\mathrm{AH}]$, we put the Young diagrams of $\mu$ and $\nu$ "back to back," separated by a vertical "wall"; thus, the diagram of $\mu+\nu$ is obtained by forgetting the wall and left-justifying the boxes. For example,

$$
(3,2,1,1)=\square \quad \text { and } \quad((2,1) ;(3,2,1,1))=\square
$$

Finally, to any partition $\lambda \in \mathcal{P}_{n}$, we associate the quantity

$$
n(\lambda)=\sum_{i=1}^{\infty}(i-1) \lambda_{i}=\sum_{i=1}^{\infty}\left(\begin{array}{c}
\left(\lambda^{\mathbf{t}}\right)_{i} \\
2
\end{array}\right)
$$

\subsection{Signed partitions}

A signed partition is a pair $(\lambda, \epsilon)$, where $\lambda$ is a partition and $\epsilon:\{1, \ldots$, $\ell(\lambda)\} \rightarrow\{+,-\}$ is a function such that if $\lambda_{i}=\lambda_{j}, \epsilon(i)=+$, and $\epsilon(j)=-$ hold, then $i<j$. A signed partition determines two subordinate partitions $\lambda^{(+)}$and $\lambda^{(-)}$as follows. Define compositions $\lambda^{(+)}$and $\widetilde{\lambda}^{(-)}$by

$$
\lambda_{i}^{(+)}=\left\{\begin{array}{ll}
\left\lceil\lambda_{i} / 2\right\rceil & \text { if } \epsilon(i)=+, \\
\left\lfloor\lambda_{i} / 2\right\rfloor & \text { if } \epsilon(i)=-,
\end{array} \quad \widetilde{\lambda}_{i}^{(-)}=\lambda_{i}-\lambda_{i}^{(+)} .\right.
$$

Then $\lambda^{(+)}$is a partition, and $\widetilde{\lambda}^{(-)}$fails to be a partition exactly when there exist some $i<j$ such that $\lambda_{i}=\lambda_{j}$ is odd, $\epsilon(i)=+$, and $\epsilon(j)=-$. We define $\lambda^{(-)}$to be the partition obtained by rearranging the parts of $\tilde{\lambda}^{(-)}$ in decreasing order. The signature of a signed partition $(\lambda, \epsilon)$ is the pair of 
integers $\left(\left|\lambda^{(+)}\right|,\left|\lambda^{(-)}\right|\right)$. The set of all signed partitions of signature $\left(d, d^{\prime}\right)$ is denoted $\mathcal{S P} d, d^{\prime}$.

The visual interpretation is as follows. The signed partition $(\lambda, \epsilon)$ may be drawn as the Young diagram of $\lambda$ with the values of $\epsilon$ filled in along the first column and then signs inserted in the rest of the diagram so that + and alternate across rows. The condition on $\epsilon$ stipulates that among the rows of a certain length, those beginning with + come above those beginning with - . For instance, $((6,3,3,3,2,1),(-,+,-,-,-,+))$ would be drawn as

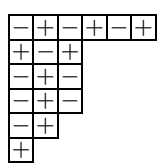

Then, $\lambda^{(+)}$is obtained by erasing all - boxes and left-justifying the remaining boxes, and $\lambda^{(-)}$is defined analogously but possibly with the additional step of reordering the rows. In our example, we have

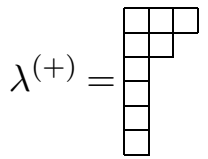

and

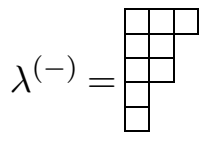

The signature of $(\lambda, \epsilon)$ simply counts the + boxes and the - boxes.

\subsection{Signed quasi-bipartitions}

A quasi-partition is a composition $\lambda=\left(\lambda_{1}, \lambda_{2}, \ldots\right)$ satisfying $\lambda_{i} \geq \lambda_{j}-1$ whenever $i \leq j$.

A signed quasi-bipartition is a triple $(\mu ; \nu, \epsilon)$ where $\mu$ and $\nu$ are quasipartitions such that $\mu+\nu$ is a partition, and $(\mu+\nu, \epsilon)$ is a signed partition such that

$$
\epsilon(i)= \begin{cases}+ & \text { if } \mu_{i} \geq 1 \text { is odd } \\ - & \text { if } \mu_{i} \geq 2 \text { is even, } \\ - & \text { if } \mu_{i}=0 \text { and there is some } j<i \text { such that } \nu_{j}=\nu_{i}-1 \\ & \text { or } \mu_{i}=0 \text { and there is some } j>i \text { such that } \mu_{j}=1 .\end{cases}
$$

Note that we do not specify $\epsilon(i)$ if $\mu_{i}=0$, and there is no $j$ as above. The signature of $(\mu ; \nu, \epsilon)$ is the signature of $(\mu+\nu, \epsilon)$. The set of all signed quasi-bipartitions of signature $\left(d, d^{\prime}\right)$ is denoted $\mathcal{S} \mathcal{Q}_{d, d^{\prime}}$. 
REMARK 2.1. The definition of signed quasi-bipartition given here is equivalent to that of "striped 2-bipartition" given by Johnson [J, Definition 4.1]. The main difference is that where we would have $\mu_{i}=0, \nu_{i}=$ $s \geq 1$, and $\epsilon(i)=+$, Johnson would have $\mu_{i}=-1, \nu_{i}=s+1$, and $\epsilon(i)=+$. The restrictions we imposed on this case are equivalent to saying that the quasi-partition inequalities continue to hold if one applies this shift. Johnson's convention achieves a uniform rule that $\epsilon(i)=+$ if and only if $\mu_{i}$ is odd, but at the cost of allowing $\mu$ to have negative parts.

As above, we can draw a signed quasi-bipartition $(\mu ; \nu, \epsilon)$ as a pair of backto-back diagrams of boxes with the values of $\epsilon$ entered in the leftmost box of each row and with + and - alternating across rows. The condition on $\epsilon$ implies that every box immediately to the left of the wall contains a + . For instance, the signed quasi-bipartition $((2,3,2,1,2,0,1) ;(4,2,3,3,1,2),(-,+$, $-,+,-,-,+))$ would be

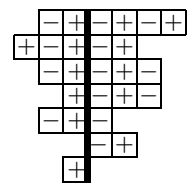

Given a signed quasi-bipartition $(\mu ; \nu, \epsilon)$ with $\mu+\nu=\lambda$, we will define subordinate bipartitions $\left(\mu^{(+)} ; \nu^{(+)}\right)$and $\left(\mu^{(-)} ; \nu^{(-)}\right)$such that

$$
\mu^{(+)}+\nu^{(+)}=\lambda^{(+)} \quad \text { and } \quad \mu^{(-)}+\nu^{(-)}=\lambda^{(-)} .
$$

We first define quasi-partitions $\widetilde{\mu}^{(+)}, \widetilde{\nu}^{(+)}, \widetilde{\mu}^{(-)}, \widetilde{\nu}^{(-)}$which count the number of boxes of a given sign on a given side of the wall and in a given row:

$$
\begin{array}{ll}
\widetilde{\mu}_{i}^{(+)}=\left\lceil\mu_{i} / 2\right\rceil ; & \widetilde{\mu}_{i}^{(-)}=\mu_{i}-\widetilde{\mu}_{i}^{(+)} ; \\
\widetilde{\nu}_{i}^{(+)}=\left\{\begin{array}{lll}
\left\lceil\nu_{i} / 2\right\rceil & \text { if } \mu_{i}=0 \text { and } \epsilon(i)=+, & \widetilde{\nu}_{i}^{(-)}=\nu_{i}-\widetilde{\nu}_{i}^{(+)} . \\
\left\lfloor\nu_{i} / 2\right\rfloor & \text { otherwise; }
\end{array}\right.
\end{array}
$$

Let $\lambda$ be the partition $\mu+\nu$. Then $\widetilde{\mu}^{(+)}+\widetilde{\nu}^{(+)}=\lambda^{(+)}$is a partition, but $\widetilde{\mu}^{(-)}+\widetilde{\nu}^{(-)}=\widetilde{\lambda}^{(-)}$may not be, as seen above. If necessary, apply some permutation simultaneously to the parts of $\widetilde{\mu}^{(-)}$and to the parts of $\widetilde{\nu}^{(-)}$ so that $\widetilde{\mu}^{(-)}+\widetilde{\nu}^{(-)}$becomes the partition $\lambda^{(-)}$; it is easy to see that $\widetilde{\mu}^{(-)}$ and $\widetilde{\nu}^{(-)}$will still be quasi-partitions after this permutation. For example, 
starting from the above signed quasi-bipartition, we obtain

$$
\left(\widetilde{\mu}^{(+)} ; \widetilde{\nu}^{(+)}\right)=\underbrace{}_{\square} \quad \text { and } \quad\left(\widetilde{\mu}^{(-)} ; \widetilde{\nu}^{(-)}\right)=\frac{\square}{\square}
$$

by deleting all - boxes and all + boxes, respectively. To produce a bipartition $\left(\mu^{(+)} ; \nu^{(+)}\right)$from $\left(\widetilde{\mu}^{(+)} ; \widetilde{\nu}^{(+)}\right)$, we apply the rectification procedure of [AH, Lemma 2.4], which in the context of quasi-partitions means that

$$
\mu_{i}^{(+)}= \begin{cases}\widetilde{\mu}_{i}^{(+)}+1 & \text { if } \widetilde{\mu}_{j}^{(+)}=\widetilde{\mu}_{i}^{(+)}+1 \text { for some } j>i \\ \widetilde{\mu}_{i}^{(+)} & \text {or } \widetilde{\nu}_{j}^{(+)}=\widetilde{\nu}_{i}^{(+)}-1 \text { for some } j<i\end{cases}
$$

and $\nu_{i}^{(+)}=\lambda_{i}^{(+)}-\mu_{i}^{(+)}$. We obtain $\left(\mu^{(-)} ; \nu^{(-)}\right)$from $\left(\widetilde{\mu}^{(-)} ; \widetilde{\nu}^{(-)}\right)$by the same rule. In our example, we have

$$
\left(\mu^{(+)} ; \nu^{(+)}\right)=\sqrt{H} \quad \text { and } \quad\left(\mu^{(-)} ; \nu^{(-)}\right)=+
$$

\subsection{Nilpotent orbits and enhanced nilpotent orbits}

Let $V$ be a complex vector space of dimension $d$, and let

$$
\mathcal{N}_{V}=\{x \in \operatorname{End}(V) \mid x \text { is nilpotent }\} .
$$

(As in the introduction, we may omit the subscript from $\mathcal{N}_{V}$ if only one vector space is involved.) Here $\mathrm{GL}(V)$ acts on $\mathcal{N}_{V}$ by conjugation. The Jordan form theorem gives us the following well-known parameterization of orbits by partitions. For $x \in \mathcal{N}_{V}$ and $\lambda \in \mathcal{P}_{d}$, a basis of $V$ is said to be a Jordan basis for $x$ of type $\lambda$ if the elements of the basis can be identified with the boxes in the diagram of $\lambda$, in such a way that $x$ sends a given box to the box on its left, or to zero if there is no box on its left.

Lemma 2.2. The GL $(V)$-orbits on $\mathcal{N}_{V}$ are in bijection with $\mathcal{P}_{d}$. For $\lambda \in$ $\mathcal{P}_{d}$, the corresponding orbit $\mathcal{O}_{\lambda}$ consists of all $x$ for which there is a Jordan basis of type $\lambda$.

If $x \in \mathcal{O}_{\lambda}$, we refer to $\lambda$ as the Jordan type of $x$. 
The following characterization of the closures of these orbits is also well known. Recall the dominance partial order on partitions: if $\rho, \lambda \in \mathcal{P}_{d}$, then $\rho \leq \lambda$ if and only if for all $k$,

$$
\sum_{i=1}^{k} \rho_{i} \leq \sum_{i=1}^{k} \lambda_{i}
$$

Lemma 2.3. For a nilpotent endomorphism $x \in \mathcal{N}_{V}$, the following conditions are equivalent:

(1) $x \in \overline{\mathcal{O}_{\lambda}}$;

(2) the Jordan type $\rho$ of $x$ satisfies $\rho \leq \lambda$;

(3) $V$ admits a filtration $0=V_{0} \subset V_{1} \subset \cdots \subset V_{\lambda_{1}}=V$ such that $x\left(V_{i}\right) \subset V_{i-1}$ and $\operatorname{dim} V_{i} / V_{i-1}=\lambda_{\lambda_{1}+1-i}^{\mathbf{t}}$

(4) $V$ admits a filtration $0=V_{0} \subset V_{1} \subset \cdots \subset V_{\lambda_{1}}=V$ such that $x\left(V_{i}\right) \subset V_{i-1}$ and $\operatorname{dim} V_{i} / V_{i-1}=\lambda_{i}^{\mathbf{t}}$.

Next, the enhanced nilpotent cone associated to $V$ is the variety $V \times \mathcal{N}_{V}$. The group $\mathrm{GL}(V)$ acts on this cone with finitely many orbits as well. For $(v, x) \in V \times \mathcal{N}_{V}$ and $(\mu ; \nu) \in \mathcal{Q}_{d}$, a basis of $V$ is said to be a normal basis for $(v, x)$ of type $(\mu ; \nu)$ if the elements of the basis can be identified with the boxes in the diagram of $(\mu ; \nu)$, in such a way that $x$ sends a given box to the box on its left, or to zero if there is no box on its left; and $v$ is the sum of the boxes immediately left of the wall.

Lemma 2.4 ([AH, Proposition 2.3], [T, Theorem 1]). The GL(V)-orbits on $V \times \mathcal{N}_{V}$ are in bijection with $\mathcal{Q}_{d}$. For $(\mu ; \nu) \in \mathcal{Q}_{d}$, the corresponding orbit $\mathcal{O}_{\mu ; \nu}$ consists of those $(v, x)$ for which there is a normal basis of type $(\mu ; \nu)$.

If $(v, x) \in \mathcal{O}_{\mu ; \nu}$, we will refer to $(\mu ; \nu)$ as the type of $(v, x)$.

From this description, it is clear that the projection map $\bar{\pi}^{V}: V \times \mathcal{N}_{V} \rightarrow$ $\mathcal{N}_{V}$ satisfies

$$
\bar{\pi}^{V}\left(\mathcal{O}_{\mu ; \nu}\right)=\mathcal{O}_{\mu+\nu}
$$

Note that we can identify the ordinary nilpotent cone $\mathcal{N}_{V}$ with the closed subvariety $\{0\} \times \mathcal{N}_{V}$ of the enhanced nilpotent cone $V \times \mathcal{N}_{V}$. Under this identification, the orbit $\mathcal{O}_{\lambda} \subset \mathcal{N}_{V}$ corresponds to the orbit $\mathcal{O}_{\varnothing ; \lambda} \subset V \times \mathcal{N}_{V}$. Thus, all our statements about enhanced nilpotent orbits and their closures will include a (usually well-known) statement about the unenhanced case. 
To state the analogue of Lemma 2.3, we need the partial order on $\mathcal{Q}_{d}$ defined as follows: $(\rho ; \sigma) \leq(\mu ; \nu)$ if and only if for all $k \geq 0$,

$$
\sum_{i=1}^{k}\left(\rho_{i}+\sigma_{i}\right) \leq \sum_{i=1}^{k}\left(\mu_{i}+\nu_{i}\right)
$$

and

$$
\sum_{i=1}^{k}\left(\rho_{i}+\sigma_{i}\right)+\rho_{k+1} \leq \sum_{i=1}^{k}\left(\mu_{i}+\nu_{i}\right)+\mu_{k+1} .
$$

Lemma 2.5 ([AH, Theorem 3.9, Corollary 3.4]). For $(v, x) \in V \times \mathcal{N}_{V}$, the following conditions are equivalent:

(1) $(v, x) \in \overline{\mathcal{O}_{\mu ; \nu}}$;

(2) the type $(\rho ; \sigma)$ of $(v, x)$ satisfies $(\rho ; \sigma) \leq(\mu ; \nu)$;

(3) there is an $x$-stable $|\mu|$-dimensional subspace $W \subset V$ containing $v$ such that

(a) the Jordan type $\bar{\mu}$ of $\left.x\right|_{W}$ satisfies $\bar{\mu} \leq \mu$, and

(b) the Jordan type $\bar{\nu}$ of $\left.x\right|_{V / W}$ satisfies $\bar{\nu} \leq \nu$.

Here, and subsequently, when $x$ is a nilpotent endomorphism of $V$ and $W$ is an $x$-stable subspace, $\left.x\right|_{W}$ and $\left.x\right|_{V / W}$ denote the induced nilpotent endomorphisms of $W$ and of $V / W$.

\subsection{Covering relations}

We have seen in Lemmas 2.3 and 2.5 that the inclusion relations among ordinary (resp., enhanced) nilpotent orbit closures correspond to a combinatorial partial order on the set of partitions (resp., bipartitions). For later use, we recall the covering relations which generate these partial orders. Geometrically, these covering relations correspond to minimal degenerations of orbits.

It is well known that the covering relations $\lambda^{\prime}<\lambda$ in the dominance partial order on $\mathcal{P}_{d}$ are those in which a single box in the diagram for $\lambda$ moves down from an outside corner to the first available inside corner, resulting in the diagram of $\lambda^{\prime}$ :

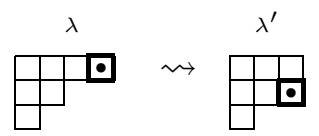

It is proved in [AH, Lemma 3.7] that there are four types of covering relations which generate the partial order on $\mathcal{Q}_{d}$. We recall the pictorial 
description of these covering relations, putting the diagram of a bipartition $(\mu ; \nu)$ on the left and the diagram of $\left(\mu^{\prime} ; \nu^{\prime}\right)<(\mu ; \nu)$ on the right. In type $(1)$, a single box moves down on the $\mu$ side of the dividing line, from an outside corner to the first available inside corner, there being no inside or outside corners on the $\nu$ side between these two positions:

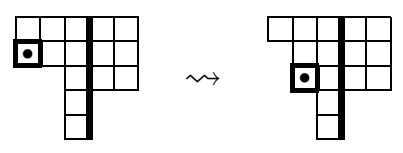

Type (2) is analogous but with the box moving on the $\nu$ side of the dividing line:

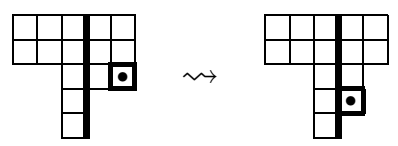

In type (3), a column of boxes (possibly a single box) moves directly to the right, from an outside corner on the $\mu$ side to an inside corner on the $\nu$ side:

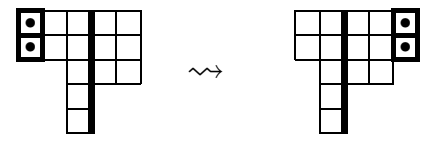

In type (4), a column of boxes (possibly a single box) moves to the left and down one row, from an outside corner on the $\nu$ side to an inside corner on the $\mu$ side:

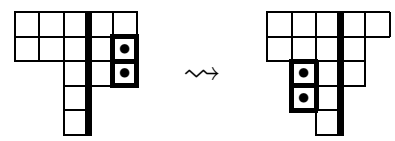

\subsection{Nilpotent pairs and enhanced nilpotent pairs}

Now, let $V$ and $V^{\prime}$ be complex vector spaces, say, of dimensions $d, d^{\prime}$, and let

$$
\mathcal{N}_{V, V^{\prime}}=\left\{(x, y) \in \operatorname{Hom}\left(V, V^{\prime}\right) \times \operatorname{Hom}\left(V^{\prime}, V\right) \mid x y \text { is nilpotent }\right\} .
$$

Note that $x y$ is nilpotent if and only if $y x$ is nilpotent, so we will make no distinction between $\mathcal{N}_{V, V^{\prime}}$ and $\mathcal{N}_{V^{\prime}, V}$. Elements $(x, y) \in \mathcal{N}_{V, V^{\prime}}$ are known as nilpotent pairs.

The group $\mathrm{GL}(V) \times \mathrm{GL}\left(V^{\prime}\right)$ acts on $\mathcal{N}_{V, V^{\prime}}$ with finitely many orbits, described as follows. For $(x, y) \in \mathcal{N}_{V, V^{\prime}}$ and $(\lambda, \epsilon) \in \mathcal{S} \mathcal{P}_{d, d^{\prime}}$, a basis of $V \oplus V^{\prime}$ is said to be a Jordan basis for $(x, y)$ of type $(\lambda, \epsilon)$ if the elements of the basis can be identified with the boxes in the diagram of $(\lambda, \epsilon)$, with the + 
boxes forming a basis of $V$ and the - boxes forming a basis of $V^{\prime}$, in such a way that $x$ sends a given + box to the - box on its left, or to zero if there is no box on its left, and $y$ sends a given - box to the + box on its left, or to zero if there is no box on its left.

Lemma 2.6 ([KP1, Section 4]). The $\left(\mathrm{GL}(V) \times \mathrm{GL}\left(V^{\prime}\right)\right)$-orbits in $\mathcal{N}_{V, V^{\prime}}$ are in bijection with $\mathcal{S} \mathcal{P}_{d, d^{\prime}}$. For $(\lambda, \epsilon) \in \mathcal{S P}_{d, d^{\prime}}$, the corresponding orbit $\mathcal{C}_{\lambda, \epsilon}$ consists of those $(x, y)$ for which there is a Jordan basis of type $(\lambda, \epsilon)$.

Consider the maps $\bar{p}_{V}^{V, V^{\prime}}: \mathcal{N}_{V, V^{\prime}} \rightarrow \mathcal{N}_{V}$ and $\bar{p}_{V^{\prime}}^{V, V^{\prime}}: \mathcal{N}_{V, V^{\prime}} \rightarrow \mathcal{N}_{V^{\prime}}$ given by

$$
\bar{p}_{V}^{V, V^{\prime}}(x, y)=y x \quad \text { and } \quad \bar{p}_{V^{\prime}}^{V, V^{\prime}}(x, y)=x y .
$$

Recall that the signed partition $(\lambda, \epsilon)$ determines subordinate partitions $\lambda^{(+)}$ and $\lambda^{(-)}$. Using the Jordan basis from Lemma 2.6, it is easy to see that

$$
\bar{p}_{V}^{V, V^{\prime}}\left(\mathcal{C}_{\lambda, \epsilon}\right)=\mathcal{O}_{\lambda^{(+)}} \quad \text { and } \quad \bar{p}_{V^{\prime}}^{V, V^{\prime}}\left(\mathcal{C}_{\lambda, \epsilon}\right)=\mathcal{O}_{\lambda(-)}
$$

Next, we consider the variety $V \times \mathcal{N}_{V, V^{\prime}}$, known as the variety of enhanced nilpotent pairs. (Note that this definition is asymmetric in $V$ and $V^{\prime}$.) The group $\mathrm{GL}(V) \times \mathrm{GL}\left(V^{\prime}\right)$ acts on this variety with finitely many orbits. These have the following parameterization due to Johnson (recall Remark 2.1), combining aspects of Lemmas 2.4 and 2.6. For $(v, x, y) \in V \times \mathcal{N}_{V, V^{\prime}}$ and $(\mu ; \nu, \epsilon) \in \mathcal{S} \mathcal{Q}_{d, d^{\prime}}$, a basis of $V \oplus V^{\prime}$ is said to be a normal basis for $(v, x, y)$ of type $(\mu ; \nu, \epsilon)$ if the elements of the basis can be identified with the boxes in the diagram of $(\mu ; \nu, \epsilon)$, with the + boxes forming a basis of $V$ and the boxes forming a basis of $V^{\prime}$, in such a way that $x$ and $y$ move boxes to the left as in the definition of a Jordan basis, and $v$ is the sum of the boxes immediately left of the wall. (Recall that by definition every box immediately left of the wall contains $\mathrm{a}+$.)

Lemma 2.7 ([J, Corollary 4.13]). The $\left(\mathrm{GL}(V) \times \mathrm{GL}\left(V^{\prime}\right)\right)$-orbits in $V \times$ $\mathcal{N}_{V, V^{\prime}}$ are in bijection with $\mathcal{S} \mathcal{Q}_{d, d^{\prime}}$. For $(\mu ; \nu, \epsilon) \in \mathcal{S} \mathcal{Q}_{d, d^{\prime}}$, the corresponding orbit $\mathcal{C}_{\mu ; \nu, \epsilon}$ consists of those $(v, x, y)$ for which there is a normal basis of type $(\mu ; \nu, \epsilon)$.

We have maps $p_{V}^{V, V^{\prime}}: V \times \mathcal{N}_{V, V^{\prime}} \rightarrow V \times \mathcal{N}_{V}$ and $p_{V^{\prime}}^{V, V^{\prime}}: V \times \mathcal{N}_{V, V^{\prime}} \rightarrow V^{\prime} \times$ $\mathcal{N}_{V^{\prime}}$ given by

$$
p_{V}^{V, V^{\prime}}(v, x, y)=(v, y x) \quad \text { and } \quad p_{V^{\prime}}^{V, V^{\prime}}(v, x, y)=(x v, x y) .
$$


We also have the map $\pi^{V, V^{\prime}}: V \times \mathcal{N}_{V, V^{\prime}} \rightarrow \mathcal{N}_{V, V^{\prime}}$ given by projection onto the second factor. These maps have the expected compatibilities:

$$
\begin{aligned}
& p_{V}^{V, V^{\prime}}\left(\mathcal{C}_{\mu ; \nu, \epsilon}\right)=\mathcal{O}_{\mu}(+) ; \nu^{(+)}, \\
& p_{V^{\prime}}^{V, V^{\prime}}\left(\mathcal{C}_{\mu ; \nu, \epsilon}\right)=\mathcal{O}_{\mu(-) ; \nu}(-), \\
& \pi^{V, V^{\prime}}\left(\mathcal{C}_{\mu ; \nu, \epsilon}\right)=\mathcal{C}_{\mu+\nu ; \epsilon} .
\end{aligned}
$$

Of course, one could consider enhanced nilpotent pair orbits in $V^{\prime} \times \mathcal{N}_{V, V^{\prime}}$ instead and thus define maps $p_{V^{\prime}}^{V^{\prime}, V}, p_{V}^{V^{\prime}, V}$, and $\pi^{V^{\prime}, V}$. In this case, one must remember to reverse the meaning of the signs, so that the + label is associated with $V^{\prime}$ and the - label with $V$.

\subsection{Orbit dimensions}

Let $\lambda \in \mathcal{P}_{d}$. The dimension of the $\mathrm{GL}(V)$-orbit $\mathcal{O}_{\lambda} \subset \mathcal{N}_{V}$ is given by the following well-known formula:

$$
\operatorname{dim} \mathcal{O}_{\lambda}=d^{2}-d-2 n(\lambda)
$$

Next, let $(\mu ; \nu) \in \mathcal{Q}_{d}$. From [AH, Proposition 2.8], we have

$$
\operatorname{dim} \mathcal{O}_{\mu ; \nu}=d^{2}-d-2 n(\mu+\nu)+|\mu|=\operatorname{dim} \bar{\pi}^{V}\left(\mathcal{O}_{\mu ; \nu}\right)+|\mu| .
$$

Moreover, the "extra" term $|\mu|$ has the following interpretation: for a point $(v, x) \in \mathcal{O}_{\mu ; \nu}$, let

$$
E^{x}=\{a \in \operatorname{End}(V) \mid a x=x a\} .
$$

Then the subspace $E^{x} v \subset V$ has dimension $|\mu|$.

LEMMA 2.8. If $\mathcal{O}_{\rho ; \sigma} \subset \overline{\mathcal{O}_{\mu ; \nu}}$, then we have

$$
\begin{gathered}
\operatorname{dim} \mathcal{O}_{\rho ; \sigma}+|\rho| \leq \operatorname{dim} \mathcal{O}_{\mu ; \nu}+|\mu|, \\
|\rho|-n(\rho+\sigma) \leq|\mu|-n(\mu+\nu) .
\end{gathered}
$$

Proof. The two inequalities are equivalent to one another by (2.6). (The difference between the left- and right-hand sides in the first statement is double that in the second statement.) It suffices to prove this in the case where $\mathcal{O}_{\rho ; \sigma}$ is a minimal degeneration of $\mathcal{O}_{\mu ; \nu}$. Recall that the minimal degenerations of enhanced nilpotent orbits were given in Section 2.5 in terms of four kinds of "moves" applied to the bipartition $(\mu ; \nu)$. Assume that $(\rho ; \sigma)$ 
is obtained from $(\mu ; \nu)$ by such a move. If the move is of type $(1)$ or $(2)$, we have $|\rho|=|\mu|$, so the first inequality holds trivially. In a move of type (3), we have $\rho+\sigma=\mu+\nu$ and $|\rho|<|\mu|$, so the second inequality holds. In a move of type (4), we have $\rho_{i}+\sigma_{i-1}=\mu_{i}+\nu_{i-1}$ for all $i$ (interpreting $\sigma_{0}$ and $\nu_{0}$ as zero). Since

$$
|\rho|-n(\rho+\sigma)=\sum_{i=1}^{\infty}(2-i)\left(\rho_{i}+\sigma_{i-1}\right)
$$

and likewise for $(\mu ; \nu)$, both inequalities hold with equality in this case.

REMARK 2.9. In Section 6, we will use the second inequality of Lemma 2.8 in a crucial way. To that end, we remark here that in moves of type (1) and (2), the difference between left- and right-hand sides in the second inequality is at least 1 . It is exactly 1 when a single box moves down to the row directly below. In moves of type (3), the difference is the number of boxes which move to the right. In moves of type (4), the difference is zero.

Next, for a signed partition $(\lambda, \epsilon)$, a formula for $\operatorname{dim} \mathcal{C}_{\lambda, \epsilon}$ is given in $[\mathrm{KP} 1$, Proposition 5.3]. We will not use that formula itself, but only the upper bound

$$
\operatorname{dim} \mathcal{C}_{\lambda, \epsilon} \leq \frac{1}{2}\left(\operatorname{dim} \mathcal{O}_{\lambda^{(+)}}+\operatorname{dim} \mathcal{O}_{\lambda^{(-)}}\right)+d d^{\prime}
$$

obtained by omitting a term that always takes nonpositive values. At one point, we will need the further fact that equality holds in (2.8) if and only if no rearrangement of parts is necessary in forming the subordinate partition $\lambda^{(-)}$from $(\lambda, \epsilon)$.

Lastly, consider an orbit $\mathcal{C}_{\mu ; \nu, \epsilon} \subset V \times \mathcal{N}_{V, V^{\prime}}$, and choose a point $(v, x, y) \in$ $\mathcal{C}_{\mu ; \nu, \epsilon}$. Define the set $E^{(x, y)} \subset \operatorname{End}(V) \oplus \operatorname{End}\left(V^{\prime}\right)$ by

$$
E^{(x, y)}=\left\{(a, b) \in \operatorname{End}(V) \oplus \operatorname{End}\left(V^{\prime}\right) \mid x a=b x \text { and } a y=y b\right\} .
$$

Let $E_{V}^{(x, y)}$ and $E_{V^{\prime}}^{(x, y)}$ denote the projections of $E^{(x, y)}$ to $\operatorname{End}(V)$ and to $\operatorname{End}\left(V^{\prime}\right)$, respectively. As noted in [J, Proposition 5.2], we have an analogue of $(2.6)$ :

$$
\operatorname{dim} \mathcal{C}_{\mu ; \nu, \epsilon}=\operatorname{dim} \pi^{V, V^{\prime}}\left(\mathcal{C}_{\mu ; \nu, \epsilon}\right)+\operatorname{dim} E_{V}^{(x, y)} v .
$$

A combinatorial formula for $\operatorname{dim} \mathcal{C}_{\mu ; \nu, \epsilon}$ is given in [J, Corollary 5.9]. We will not use that formula itself, but only the upper bounds in the following result. 
Lemma 2.10. Consider an enhanced nilpotent pair orbit $\mathcal{C}=\mathcal{C}_{\mu ; \nu, \epsilon} \subset V \times$ $\mathcal{N}_{V, V^{\prime}}$. For brevity, let $(\rho ; \sigma)=\left(\mu^{(+)} ; \nu^{(+)}\right)$, and let $\left(\rho^{\prime} ; \sigma^{\prime}\right)=\left(\mu^{(-)} ; \nu^{(-)}\right)$, so that

$$
\mathcal{O}_{\rho ; \sigma}=p_{V}^{V, V^{\prime}}(\mathcal{C}) \subset V \times \mathcal{N}_{V} \quad \text { and } \quad \mathcal{O}_{\rho^{\prime} ; \sigma^{\prime}}=p_{V^{\prime}}^{V, V^{\prime}}(\mathcal{C}) \subset V^{\prime} \times \mathcal{N}_{V^{\prime}}
$$

Next, let

$$
\mathcal{P}=\mathcal{O}_{\rho+\sigma}=\bar{\pi}^{V}\left(\mathcal{O}_{\rho ; \sigma}\right) \subset \mathcal{N}_{V}
$$

and let

$$
\mathcal{P}^{\prime}=\mathcal{O}_{\rho^{\prime}+\sigma^{\prime}}=\bar{\pi}^{V^{\prime}}\left(\mathcal{O}_{\rho^{\prime} ; \sigma^{\prime}}\right) \subset \mathcal{N}_{V^{\prime}}
$$

The dimension of $\mathcal{C}$ satisfies the following two inequalities:

$$
\begin{aligned}
\operatorname{dim} \mathcal{C} & \leq \operatorname{dim} \mathcal{O}_{\rho ; \sigma}+\frac{1}{2}\left(\operatorname{dim} \mathcal{P}^{\prime}-\operatorname{dim} \mathcal{P}\right)+d d^{\prime} \\
\operatorname{dim} \mathcal{C} & \leq \operatorname{dim} \mathcal{O}_{\rho^{\prime} ; \sigma^{\prime}}+\frac{1}{2}\left(\operatorname{dim} \mathcal{P}-\operatorname{dim} \mathcal{P}^{\prime}\right)+|\rho|-\left|\rho^{\prime}\right|+d d^{\prime}
\end{aligned}
$$

Proof. Let $(v, x, y) \in \mathcal{C}$. It is easy to see that $E_{V}^{(x, y)} \subset E^{y x}$, where the latter is defined as in (2.7), so that

$$
\operatorname{dim} E_{V}^{(x, y)} v \leq \operatorname{dim} E^{y x} v .
$$

By the remarks following (2.6), we have $\operatorname{dim} E^{y x} v=|\rho|$. Next, (2.8) says that $\operatorname{dim} \pi^{V, V^{\prime}}(\mathcal{C}) \leq(1 / 2)\left(\operatorname{dim} \mathcal{P}+\operatorname{dim} \mathcal{P}^{\prime}\right)+d d^{\prime}$. Combining these observations with (2.9), we obtain

$$
\operatorname{dim} \mathcal{C} \leq \frac{1}{2}\left(\operatorname{dim} \mathcal{P}+\operatorname{dim} \mathcal{P}^{\prime}\right)+d d^{\prime}+|\rho|
$$

Recall from (2.6) that $\operatorname{dim} \mathcal{O}_{\rho ; \sigma}=\operatorname{dim} \mathcal{P}+|\rho|$ and that $\operatorname{dim} \mathcal{O}_{\rho^{\prime} ; \sigma^{\prime}}=\operatorname{dim} \mathcal{P}^{\prime}+$ $\left|\rho^{\prime}\right|$. Both inequalities in the lemma follow.

\section{§3. Invariant theory for enhanced nilpotent orbits and pairs}

In this section, we fix two vector spaces $V$ and $V^{\prime}$ such that $\operatorname{dim} V^{\prime}>$ $\operatorname{dim} V$. Let $r=\operatorname{dim} V^{\prime}-\operatorname{dim} V$. Given an enhanced nilpotent orbit closure $\overline{\mathcal{O}_{\mu ; \nu}} \subset V \times \mathcal{N}_{V}$, there are two natural ways to construct from it a subvariety of the enhanced nilpotent cone $V^{\prime} \times \mathcal{N}_{V^{\prime}}$; namely, we can form either $p_{V^{\prime}}^{V, V^{\prime}}\left(\left(p_{V}^{V, V^{\prime}}\right)^{-1}\left(\overline{\mathcal{O}_{\mu ; \nu}}\right)\right)$ or $p_{V^{\prime}}^{V^{\prime}, V}\left(\left(p_{V}^{V^{\prime}, V}\right)^{-1}\left(\overline{\mathcal{O}_{\mu ; \nu}}\right)\right)$, using the appropriate one of the following diagrams:

$$
V \times \mathcal{N}_{V} \stackrel{p_{V}^{V, V^{\prime}}}{\longleftarrow} V \times \mathcal{N}_{V, V^{\prime}} \stackrel{p_{V^{\prime}}^{V, V^{\prime}}}{\longrightarrow} V^{\prime} \times \mathcal{N}_{V^{\prime}},
$$




$$
V \times \mathcal{N}_{V} \stackrel{p_{V}^{V^{\prime}, V}}{\longleftarrow} V^{\prime} \times \mathcal{N}_{V, V^{\prime}} \stackrel{p_{V^{\prime}}^{V^{\prime}, V}}{\longrightarrow} V^{\prime} \times \mathcal{N}_{V^{\prime}} .
$$

(This use of "I" and "I" in the labels will be compatible with notation to be introduced in Section 4.) The goal of this section (see Proposition 3.4) is to identify these subvarieties of $V^{\prime} \times \mathcal{N}_{V^{\prime}}$. We begin with the following result, an enhanced analogue of [KP1, Theorem 2.2].

Here, and throughout the paper, we write $H \backslash X X$ for Spec $\mathbb{C}[X]^{H}$, where $X$ is an affine variety acted on by a reductive group $H$. Since we are working over $\mathbb{C}$, an $H$-equivariant closed embedding $X \hookrightarrow Y$ induces a closed embedding $H \backslash X \hookrightarrow H \backslash \backslash Y$.

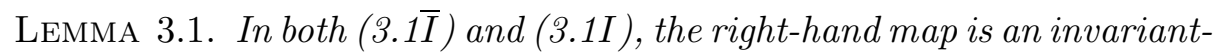
theoretic $\mathrm{GL}(V)$-quotient map onto its image. That is, $p_{V^{\prime}}^{V, V^{\prime}}$ induces an isomorphism

$$
\mathrm{GL}(V) \backslash\left(V \times \mathcal{N}_{V, V^{\prime}}\right) \stackrel{\sim}{\rightarrow} p_{V^{\prime}}^{V, V^{\prime}}\left(V \times \mathcal{N}_{V, V^{\prime}}\right)
$$

and $p_{V^{\prime}}^{V^{\prime}, V}$ induces an isomorphism

$$
\mathrm{GL}(V) \backslash\left(V^{\prime} \times \mathcal{N}_{V, V^{\prime}}\right) \stackrel{\sim}{\rightarrow} p_{V^{\prime}}^{V^{\prime}, V}\left(V^{\prime} \times \mathcal{N}_{V, V^{\prime}}\right) .
$$

Proof. A fundamental result of invariant theory states that for three vector spaces $U, U^{\prime}, U^{\prime \prime}$, the composition map $m: \operatorname{Hom}\left(U, U^{\prime}\right) \times \operatorname{Hom}\left(U^{\prime}, U^{\prime \prime}\right) \rightarrow$ $\operatorname{Hom}\left(U, U^{\prime \prime}\right)$ induces a closed embedding

$$
\operatorname{GL}\left(U^{\prime}\right) \backslash\left(\operatorname{Hom}\left(U, U^{\prime}\right) \times \operatorname{Hom}\left(U^{\prime}, U^{\prime \prime}\right)\right) \hookrightarrow \operatorname{Hom}\left(U, U^{\prime \prime}\right) .
$$

See, for instance, [D, Proposition 1.4c], which also shows that the image of this embedding consists of those linear maps in $\operatorname{Hom}\left(U, U^{\prime \prime}\right)$ whose rank is at most $\operatorname{dim} U^{\prime}$. Hence, for any (reduced) $\operatorname{GL}\left(U^{\prime}\right)$-stable closed subvariety $Y \subset$ $\operatorname{Hom}\left(U, U^{\prime}\right) \times \operatorname{Hom}\left(U^{\prime}, U^{\prime \prime}\right)$, we get an isomorphism $\operatorname{GL}\left(U^{\prime}\right) \backslash Y \stackrel{\sim}{\rightarrow} m(Y)$.

From this, (3.3) follows by taking $U=U^{\prime \prime}=V^{\prime}, U^{\prime}=V$, and $Y=\mathcal{N}_{V, V^{\prime}}$, since $\mathrm{GL}(V)$ acts trivially on $V^{\prime}$. We also get a description of the image:

$$
p_{V^{\prime}}^{V^{\prime}, V}\left(V^{\prime} \times \mathcal{N}_{V, V^{\prime}}\right)=V^{\prime} \times\left\{z \in \mathcal{N}_{V^{\prime}} \mid \operatorname{dim}(\operatorname{im}(z)) \leq \operatorname{dim} V\right\}
$$

For $(3.2)$, let $U=\mathbb{C} \times V^{\prime}$, let $U^{\prime}=V$, and let $U^{\prime \prime}=V^{\prime}$. Then the result follows using the identifications

$$
\operatorname{Hom}\left(U, U^{\prime}\right) \cong V \times \operatorname{Hom}\left(V^{\prime}, V\right) \quad \text { and } \quad \operatorname{Hom}\left(U, U^{\prime \prime}\right) \cong V^{\prime} \times \operatorname{End}\left(V^{\prime}\right) .
$$


We also get a description of the image:

$$
p_{V^{\prime}}^{V, V^{\prime}}\left(V \times \mathcal{N}_{V, V^{\prime}}\right)=\left\{(w, z) \in V^{\prime} \times \mathcal{N}_{V^{\prime}} \mid \operatorname{dim}(\mathbb{C} w+\operatorname{im}(z)) \leq \operatorname{dim} V\right\}
$$

These formulas for the images of $p_{V^{\prime}}^{V^{\prime}, V}$ and $p_{V^{\prime}}^{V, V^{\prime}}$ are the $(\mu ; \nu)=(\operatorname{dim} V ; \varnothing)$ special cases of Proposition 3.4 below.

Lemma 3.2. Let $x: V \rightarrow V^{\prime}$, and let $y: V^{\prime} \rightarrow V$. Let $\alpha=\left(a_{1}, a_{2}, \ldots, a_{k}\right)$ be a composition of $\operatorname{dim} V$, and suppose that we have a filtration

$$
0=V_{0} \subset V_{1} \subset \cdots \subset V_{k}=V
$$

such that $\operatorname{dim} V_{i}=a_{1}+a_{2}+\cdots+a_{i}$, and such that $y x\left(V_{i}\right) \subset V_{i-1}$. Assume that $r \geq a_{i}$ for all $i$. Fix $m \in\{1, \ldots, k+1\}$; if $m \leq k$, assume that $a_{m} \geq$ $a_{m+1} \geq \cdots \geq a_{k}$. Then $V^{\prime}$ admits a filtration

$$
0=V_{0}^{\prime} \subset V_{1}^{\prime} \subset \cdots \subset V_{k+1}^{\prime}=V
$$

such that $x\left(V_{i}\right) \subset V_{i}^{\prime}$ and $y\left(V_{i}^{\prime}\right) \subset V_{i-1}$, and such that

$$
\operatorname{dim} V_{i}^{\prime}= \begin{cases}a_{1}+\cdots+a_{i} & \text { if } i<m, \\ a_{1}+\cdots+a_{i-1}+r & \text { if } i \geq m\end{cases}
$$

If, in addition, $r>a_{m}$, then for any vector $u^{\prime} \in y^{-1}\left(V_{m-1}\right)$, the filtration above may be chosen so that $u^{\prime} \in V_{m}^{\prime}$.

Proof. Since $y x\left(V_{i}\right) \subset V_{i-1}$, we have $x\left(V_{i}\right) \subset y^{-1}\left(V_{i-1}\right) \subset y^{-1}\left(V_{i}\right)$. Moreover,

$$
\begin{aligned}
\operatorname{dim} x\left(V_{i}\right) & \leq a_{1}+\cdots+a_{i} \\
\operatorname{dim} y^{-1}\left(V_{i-1}\right) & \geq a_{1}+\cdots+a_{i-1}+r .
\end{aligned}
$$

We will construct the spaces $V_{i}^{\prime}$ by induction on $i$. Assume that we have already constructed subspaces $V_{0}^{\prime} \subset V_{1}^{\prime} \subset \cdots \subset V_{i-1}^{\prime}$ with the desired properties. Suppose first that $i<m$. Since $x\left(V_{i-1}\right) \subset V_{i-1}^{\prime}$, we see that

$$
\operatorname{dim} x\left(V_{i}\right) /\left(x\left(V_{i}\right) \cap V_{i-1}^{\prime}\right) \leq \operatorname{dim} V_{i} / V_{i-1}=a_{i},
$$

and therefore that

$$
\operatorname{dim}\left(V_{i-1}^{\prime}+x\left(V_{i}\right)\right) \leq a_{1}+\cdots+a_{i-1}+a_{i}
$$


Since $a_{1}+\cdots+a_{i-1}+a_{i} \leq a_{1}+\cdots+a_{i-1}+r$, there exists a subspace $V_{i}^{\prime} \subset V^{\prime}$ such that

$$
V_{i-1}^{\prime}+x\left(V_{i}\right) \subset V_{i}^{\prime} \subset y^{-1}\left(V_{i-1}\right) \quad \text { and } \quad \operatorname{dim} V_{i}^{\prime}=a_{1}+\cdots+a_{i} .
$$

In the case $i=m$, we proceed as above, except that we choose $V_{m}^{\prime}$ to have dimension $a_{1}+\cdots+a_{m-1}+r$. For the last assertion of the lemma, note that

$$
\operatorname{dim}\left(V_{m-1}^{\prime}+x\left(V_{m}\right)+\mathbb{C} u^{\prime}\right) \leq a_{1}+\cdots+a_{m}+1
$$

Provided that $r>a_{m}$, we can choose $V_{m}^{\prime}$ to satisfy the stronger condition that

$$
V_{m-1}^{\prime}+x\left(V_{m}\right)+\mathbb{C} u^{\prime} \subset V_{m}^{\prime} \subset y^{-1}\left(V_{m-1}\right)
$$

and

$$
\operatorname{dim} V_{m}^{\prime}=a_{1}+\cdots+a_{m-1}+r
$$

Finally, for the case $i>m$, we now have $\operatorname{dim} V_{i-1}^{\prime}=a_{1}+\cdots+a_{i-2}+r$, so

$$
\operatorname{dim}\left(V_{i-1}^{\prime}+x\left(V_{i}\right)\right) \leq a_{1}+\cdots+a_{i-2}+r+a_{i}
$$

Since $a_{i-1} \geq a_{i}$, we may choose a subspace $V_{i}^{\prime} \subset V$ such that

$$
V_{i-1}^{\prime}+x\left(V_{i}\right) \subset V_{i}^{\prime} \subset y^{-1}\left(V_{i-1}\right) \quad \text { and } \quad \operatorname{dim} V_{i}^{\prime}=a_{1}+\cdots+a_{i-1}+r
$$

as desired.

LEMma 3.3. Let $x: V \rightarrow V^{\prime}$ and $y: V^{\prime} \rightarrow V$ be linear maps such that $y x \in \operatorname{End}(V)$ is nilpotent. Let $W \subset V$ be a subspace stable under yx. Let $\mu \in \mathcal{P}_{\operatorname{dim} W}$, let $\nu \in \mathcal{P}_{\operatorname{dim} V / W}$, and assume that

(1) the Jordan type $\bar{\mu}$ of $\left.y x\right|_{W}$ satisfies $\bar{\mu} \leq \mu$, and

(2) the Jordan type $\bar{\nu}$ of $\left.y x\right|_{V / W}$ satisfies $\bar{\nu} \leq \nu$.

If $r \geq \ell(\mu+\nu)$, then there exist $x y$-stable subspaces $W^{\prime}$ and $\widetilde{W^{\prime}}$ of $V^{\prime}$ such that

(1) we have $x(W) \subset W^{\prime} \subset \widetilde{W^{\prime}} \subset y^{-1}(W)$;

(2) $\operatorname{dim} W^{\prime}=\operatorname{dim} W$, and $\operatorname{dim} \widetilde{W^{\prime}}=\operatorname{dim} W+r$; 
(3) the Jordan types of the maps induced by $x y$ on various subquotients of $V^{\prime}$ satisfy the following inequalities:

$$
\begin{array}{ll}
\left.x y\right|_{W^{\prime}}: \leq \mu, & \left.x y\right|_{V / W^{\prime}}: \leq \nu+1^{r}, \\
\left.x y\right|_{\widetilde{W}^{\prime}}: \leq \mu+1^{r}, & \left.x y\right|_{V / \widetilde{W}^{\prime}}: \leq \nu .
\end{array}
$$

If, in addition, $r>\ell(\nu)$, then for any vector $u^{\prime} \in y^{-1}(W)$, the space $\widetilde{W^{\prime}}$ may be chosen so that $u^{\prime} \in \widetilde{W}^{\prime}$.

Proof. By Lemma 2.3, we can endow $W$ with a filtration

$$
0=V_{0} \subset V_{1} \subset \cdots \subset V_{\mu_{1}}=W
$$

where

$$
\operatorname{dim} V_{i} / V_{i-1}=\mu_{\mu_{1}+1-i}^{\mathrm{t}} \quad \text { and } \quad y x\left(V_{i}\right) \subset V_{i-1} .
$$

We can likewise endow $V / W$ with a filtration with $\nu_{1}$ terms. Let us lift this filtration to $V$ and denote its terms as follows:

$$
W=V_{\mu_{1}} \subset V_{\mu_{1}+1} \subset \cdots \subset V_{\mu_{1}+\nu_{1}}
$$

where

$$
\operatorname{dim} V_{i} / V_{i-1}=\nu_{i-\mu_{1}}^{\mathbf{t}} \quad \text { and } \quad y x\left(V_{i}\right) \subset V_{i-1} .
$$

Since $\nu_{1}^{\mathbf{t}} \geq \cdots \geq \nu_{\nu_{1}}^{\mathbf{t}}, r \geq \mu_{i}^{\mathbf{t}}$ for all $i$, and $r \geq \nu_{i}^{\mathbf{t}}$ for all $i$, we can apply Lemma 3.2 with $m=\mu_{i}+1$ to obtain a filtration

$$
0=V_{0}^{\prime} \subset V_{1}^{\prime} \subset \cdots \subset V_{\mu_{1}+\nu_{1}+1}^{\prime}=V^{\prime}
$$

such that $x\left(V_{i}\right) \subset V_{i-1}^{\prime}, y\left(V_{i}^{\prime}\right) \subset V_{i-1}$, and

$$
\operatorname{dim} V_{i}^{\prime} / V_{i-1}^{\prime}= \begin{cases}\mu_{\mu_{1}+1-i}^{\mathbf{t}} & \text { if } i \leq \mu_{1} \\ r & \text { if } i=\mu_{1}+1 \\ \nu_{i-\mu_{1}-1}^{\mathbf{t}} & \text { if } i>\mu_{1}+1\end{cases}
$$

Let $W^{\prime}=V_{\mu_{1}}^{\prime}$, and let $\widetilde{W^{\prime}}=V_{\mu_{1}+1}^{\prime}$. The Jordan-type assertions follow from Lemma 2.3, and the last statement regarding a vector $u^{\prime} \in y^{-1}(W)$ follows from the last statement of Lemma 3.2.

We deduce the following enhanced analogue of [KP1, Lemma 2.3]. 
Proposition 3.4. Consider a $\mathrm{GL}(V)$-orbit $\mathcal{O}_{\mu ; \nu} \subset V \times \mathcal{N}_{V}$. Assume that $r \geq \ell(\mu+\nu)$.

(1) In the setting of $(3.1 \bar{I})$, we have $p_{V^{\prime}}^{V, V^{\prime}}\left(\left(p_{V}^{V, V^{\prime}}\right)^{-1}\left(\overline{\mathcal{O}_{\mu ; \nu}}\right)\right)=\overline{\mathcal{O}_{\mu ; \nu+1^{r}}}$. Indeed, $p_{V^{\prime}}^{V, V^{\prime}}$ induces an isomorphism

$$
\mathrm{GL}(V) \backslash \backslash\left(p_{V}^{V, V^{\prime}}\right)^{-1}\left(\overline{\mathcal{O}_{\mu ; \nu}}\right) \stackrel{\sim}{\rightarrow} \overline{\mathcal{O}_{\mu ; \nu+1^{r}}}
$$

(2) Assume furthermore that $r>\ell(\nu)$. In the setting of (3.1I), we have $p_{V^{\prime}}^{V^{\prime}, V}\left(\left(p_{V}^{V^{\prime}, V}\right)^{-1}\left(\overline{\mathcal{O}_{\mu ; \nu}}\right)\right)=\overline{\mathcal{O}_{\mu+1^{r} ; \nu}}$. Indeed, $p_{V^{\prime}}^{V^{\prime}, V}$ induces an isomorphism

$$
\mathrm{GL}(V) \backslash\left(p_{V}^{V^{\prime}, V}\right)^{-1}\left(\overline{\mathcal{O}_{\mu ; \nu}}\right) \stackrel{\sim}{\rightarrow} \overline{\mathcal{O}_{\mu+1^{r} ; \nu}}
$$

Proof. For both parts of the proposition, the quotient statement follows from the determination of the image, by Lemma 3.1. Moreover, that lemma implies that $p_{V^{\prime}}^{V, V^{\prime}}\left(\left(p_{V}^{V, V^{\prime}}\right)^{-1}\left(\overline{\mathcal{O}_{\mu ; \nu}}\right)\right)$ and $p_{V^{\prime}}^{V^{\prime}, V}\left(\left(p_{V}^{V^{\prime}, V}\right)^{-1}\left(\overline{\mathcal{O}_{\mu ; \nu}}\right)\right)$ are closed in $V^{\prime} \times \mathcal{N}_{V^{\prime}}$.

For part $(1)$, let $(v, x, y) \in\left(p_{V}^{V, V^{\prime}}\right)^{-1}\left(\overline{\mathcal{O}_{\mu ; \nu}}\right)$. Let $W \subset V$ be the subspace obtained by invoking Lemma 2.5 for the pair $(v, y x)$. In particular, $v \in W$. Using Lemma 3.3, we find a subspace $W^{\prime} \subset V^{\prime}$ containing $x(W)$ and, in particular, the vector $x v$. The statements about Jordan type of $\left.x y\right|_{W^{\prime}}$ and $\left.x y\right|_{V / W^{\prime}}$ from that lemma, together with Lemma 2.5 , show that $(x v, x y) \in$ $\overline{\mathcal{O}_{\mu ; \nu+1^{r}}}$. Hence, $p_{V^{\prime}}^{V, V^{\prime}}\left(\left(p_{V}^{V, V^{\prime}}\right)^{-1}\left(\overline{\mathcal{O}_{\mu ; \nu}}\right)\right) \subset \overline{\mathcal{O}_{\mu ; \nu+1^{r}}}$. To prove the reverse inclusion, it suffices to show that for any $\left(v^{\prime}, z\right) \in \mathcal{O}_{\mu ; \nu+1^{r}} \subset V^{\prime} \times \mathcal{N}_{V^{\prime}}$, there exists some $(v, x, y) \in\left(p_{V}^{V, V^{\prime}}\right)^{-1}\left(\mathcal{O}_{\mu ; \nu}\right)$ such that $x v=v^{\prime}$ and $x y=z$. Since $\ell\left(\nu+1^{r}\right)=r \geq \ell(\mu), \operatorname{im}(z)$ has dimension $\operatorname{dim}\left(V^{\prime}\right)-r=\operatorname{dim} V$ and contains $v^{\prime}$. Fixing any vector space isomorphism $x: V \stackrel{\sim}{\rightarrow} \operatorname{im}(z)$, we can define $v$ and $y$ uniquely by the equations $x v=v^{\prime}$ and $x y=z$, and it is easy to see that $(v, y x) \in \mathcal{O}_{\mu ; \nu}$, as desired.

For part $(2)$, let $\left(v^{\prime}, x, y\right) \in\left(p_{V}^{V^{\prime}, V}\right)^{-1}\left(\overline{\mathcal{O}_{\mu ; \nu}}\right)$. Let $W \subset V$ be the subspace obtained by invoking Lemma 2.5 for the pair $\left(y v^{\prime}, y x\right)$, so that $y v^{\prime} \in W$. Using the fact that $r>\ell(\nu)$, we may invoke Lemma 3.3 to find a subspace $\widetilde{W^{\prime}} \subset V^{\prime}$ containing $v^{\prime}$. The statements about Jordan type of $\left.x y\right|_{\widetilde{W}^{\prime}}$ and $\left.x y\right|_{V / \widetilde{W}^{\prime}}$ from that lemma, together with Lemma 2.5, show that $\left(v^{\prime}, x y\right) \in$ $\overline{\mathcal{O}_{\mu+1^{r} ; \nu}}$. Hence, $p_{V^{\prime}}^{V^{\prime}, V}\left(\left(p_{V}^{V^{\prime}, V}\right)^{-1}\left(\overline{\mathcal{O}_{\mu ; \nu}}\right)\right) \subset \overline{\mathcal{O}_{\mu+1^{r} ; \nu}}$. To prove the reverse inclusion, it suffices to show that for any $\left(v^{\prime}, z\right) \in \mathcal{O}_{\mu+1^{r} ; \nu} \subset V^{\prime} \times \mathcal{N}_{V^{\prime}}$, there exists some $\left(v^{\prime}, x, y\right) \in\left(p_{V}^{V^{\prime}, V}\right)^{-1}\left(\mathcal{O}_{\mu ; \nu}\right)$ such that $x y=z$. Since $\ell\left(\mu+1^{r}\right)=$ $r>\ell(\nu), \operatorname{im}(z)$ has dimension $\operatorname{dim}\left(V^{\prime}\right)-r=\operatorname{dim} V$. Fixing any vector space 
isomorphism $x: V \stackrel{\sim}{\rightarrow} \operatorname{im}(z)$, we can define $y$ uniquely by the equation $x y=z$, and it is easy to see that $(y v, y x) \in \mathcal{O}_{\mu ; \nu}$, as desired.

\section{$\S 4$. Enhanced quiver varieties of type $A$}

Fix a positive integer $n$ and a bipartition $(\mu ; \nu) \in \mathcal{Q}_{n}$. Form the partition $\lambda=\mu+\nu$, and let $t=\lambda_{1}=\ell\left(\lambda^{\mathbf{t}}\right)$. That is, $t$ is the largest part of $\lambda$, and it is the number of columns in the diagram of $\lambda$. It will be convenient to refer to the lengths of these columns in increasing order, so we define

$$
0<r_{0} \leq r_{1} \leq \cdots \leq r_{t-1} \quad \text { by } \quad r_{i}=\lambda_{t-i}^{\mathbf{t}} \text {. }
$$

Now the set of columns of $\lambda$ can be viewed as the disjoint union of the set of columns of $\mu$ and the set of columns of $\nu$. We adopt the convention that among columns of the same length, those coming from $\mu$ are labeled before those coming from $\nu$. More formally, we let $I \subset\{0, \ldots, t-1\}$ be the unique subset such that the $r_{i}$ for $i \in I$ are the column lengths of $\mu$ in nondecreasing order, and $r_{i}=r_{i+1}$ and $i+1 \in I$ together imply that $i \in I$. We define $\bar{I}=\{0, \ldots, t-1\} \backslash I$, so that the $r_{i}$ for $i \in \bar{I}$ are the column lengths of $\nu$ in nondecreasing order. Thus, the sequence $\left(r_{i}\right)$ and the set $I$ together determine the bipartition $(\mu ; \nu)$.

Let $U_{i}=\mathbb{C}^{r_{0}+\cdots+r_{i-1}}$ for $0 \leq i \leq t$. Note that $U_{0}=0, U_{t}=\mathbb{C}^{n}$. It is primarily $U_{t}$ which we think of as the vector space $V$ in the definition of enhanced nilpotent orbits.

The notation and conventions of the preceding paragraph will remain in effect for the next three sections. The aim of this section is to define the enhanced quiver variety associated to these data and to prove that the normality of that variety implies the normality of $\overline{\mathcal{O}_{\mu ; \nu}}$. In the subsequent two sections, we will make progress on studying the normality of enhanced quiver varieties. Throughout, we are guided by the results of Kraft and Procesi [KP1] in the unenhanced case, which in our framework is the special case where $I=\varnothing$ (i.e., $\mu=\varnothing)$.

\subsection{Review of results of Kraft-Procesi}

We first recall the "classical" version of our variety, denoted $Z$ in [KP1]. 
Definition 4.1. Let $\Lambda_{\lambda}=\Lambda_{\left(r_{i}\right)}$ be the affine variety consisting of tuples $\left(A_{i}, B_{i}\right)$ of linear maps arranged as follows:

$$
U_{0} \underset{B_{0}}{\stackrel{A_{0}}{\rightleftarrows}} U_{1} \underset{B_{1}}{\stackrel{A_{1}}{\rightleftarrows}} U_{2} \underset{B_{2}}{\stackrel{A_{2}}{\rightleftarrows}} \cdots \underset{B_{t-2}}{\stackrel{A_{t-2}}{\rightleftarrows}} U_{t-1} \underset{B_{t-1}}{\stackrel{A_{t-1}}{\rightleftarrows}} U_{t},
$$

which satisfy the equations

$$
\left.B_{i} A_{i}=A_{i-1} B_{i-1} \text { (equation in } \operatorname{End}\left(U_{i}\right)\right) \text { for all } 1 \leq i \leq t-1 .
$$

Since $A_{0}$ and $B_{0}$ are zero maps, the first equation says that $B_{1} A_{1}=0$; it follows that $\left(A_{i-1}, B_{i-1}\right)$ is a nilpotent pair for all $1 \leq i \leq t$.

Note that the group $\prod_{i=0}^{t} \operatorname{GL}\left(U_{i}\right)$ acts on $\Lambda_{\left(r_{i}\right)}$ via

$$
\left(g_{i}\right) \cdot\left(A_{i}, B_{i}\right)=\left(g_{i+1} A_{i} g_{i}^{-1}, g_{i} B_{i} g_{i+1}^{-1}\right) .
$$

Later we will be taking a quotient by the action of $H=\prod_{i=0}^{t-1} \operatorname{GL}\left(U_{i}\right)$ but retaining the action of $\operatorname{GL}\left(U_{t}\right)=\mathrm{GL}_{n}(\mathbb{C})$; the vector space $U_{t}$ is in that sense on a different footing from the other $U_{i}$.

REMARK 4.2. In the context of quiver varieties, $\Lambda_{\left(r_{i}\right)}$ is a special case of Nakajima's variety of quadruples satisfying the Atiyah-Drinfeld-HitchinManin (ADHM) equation, where the Dynkin diagram is that of type $A_{t-1}$. In the notation of $[\mathrm{M}], \Lambda_{\left(r_{i}\right)}=\Lambda(d, v)$, where $d=(0, \ldots, 0, n)$ and $v=\left(r_{0}, r_{0}+\right.$ $\left.r_{1}, \ldots, r_{0}+\cdots+r_{t-2}\right)$.

We also introduce notation for the naively expected dimension of $\Lambda_{\left(r_{i}\right)}$, that is, the number of coordinates of the variables $A_{i}, B_{i}$ minus the number of equations in those coordinates in the definition of the variety

$$
\begin{aligned}
d_{\left(r_{i}\right)} & =2 \sum_{i=0}^{t-1}\left(\operatorname{dim} U_{i}\right)\left(\operatorname{dim} U_{i+1}\right)-\sum_{i=1}^{t-1}\left(\operatorname{dim} U_{i}\right)^{2} \\
& =\sum_{i=1}^{t-1}\left(r_{0}+\cdots+r_{i-1}\right)^{2}+2 \sum_{0 \leq i<j \leq t-1} r_{i} r_{j} .
\end{aligned}
$$

EXAMPLE 4.3. When $t=2$ (and ignoring the zero maps), the variety $\Lambda_{\left(r_{0}, r_{1}\right)}$ consists of pairs of maps

$$
U_{1} \underset{B_{1}}{\stackrel{A_{1}}{\rightleftarrows}} U_{2} \quad \text { such that } B_{1} A_{1}=0 .
$$


Note that the kernel of any $B_{1}: U_{2} \rightarrow U_{1}$ has dimension at least $\operatorname{dim} U_{2}-$ $\operatorname{dim} U_{1}=r_{1}$, and by assumption, $r_{1} \geq r_{0}=\operatorname{dim} U_{1}$. It follows that the pairs $\left(A_{1}, B_{1}\right)$ where $A_{1}$ is injective form a dense open subvariety of $\Lambda_{\left(r_{0}, r_{1}\right)}$. This open subvariety is a fiber bundle over the Grassmannian $\operatorname{Gr}_{r_{0}}\left(U_{2}\right)$, with fibers isomorphic to $\mathrm{GL}_{r_{0}}(\mathbb{C}) \times \operatorname{Hom}\left(\mathbb{C}^{r_{1}}, \mathbb{C}^{r_{0}}\right)$. So $\Lambda_{\left(r_{0}, r_{1}\right)}$ is irreducible of dimension $r_{0}^{2}+2 r_{0} r_{1}=d_{\left(r_{0}, r_{1}\right)}$.

Kraft and Procesi proved in general that $\Lambda_{\left(r_{i}\right)}=\Lambda_{\lambda}$ is not just irreducible.

Theorem 4.4 ([KP1, Theorem 3.3]). $\Lambda_{\left(r_{i}\right)}$ is a normal complete intersection of dimension $d_{\left(r_{i}\right)}$.

REMARK 4.5. The conventions imposed at the beginning of the section imply that $r_{0} \leq r_{1} \leq \cdots \leq r_{t-1}$, and the theorem depends on this assumption. If the $r_{i}$ are not weakly increasing, $\Lambda_{\left(r_{i}\right)}$ may still be defined as above, but it may not even be irreducible, let alone normal.

\subsection{Enhanced quiver varieties}

Now we "enhance" $\Lambda_{\left(r_{i}\right)}$ by incorporating vectors which are related by the linear maps, in a way determined by the subset $I \subset\{0, \ldots, t-1\}$. Roughly, the idea is that each nilpotent pair $\left(A_{i}, B_{i}\right)$ in the definition of $\Lambda_{\left(r_{i}\right)}$ should be replaced by an enhanced nilpotent pair; the question is which of the vector spaces $U_{i}$ and $U_{i+1}$ should be distinguished as the one containing the vector. Proposition 3.4 tells us that if $i \in \bar{I}$, meaning that the corresponding column belongs to the $\nu$ side of the bipartition, then the vector should belong to $U_{i}$; and if $i \in I$, meaning that the corresponding column belongs to the $\mu$ side of the bipartition, then the vector should belong to $U_{i+1}$. In each case we obtain a vector in the other vector space by applying the appropriate map. There is then a natural consistency condition when the enhanced nilpotent pairs are assembled together, resulting in the following definition.

Definition 4.6. Let $\Lambda_{\mu ; \nu}=\Lambda_{\left(r_{i}\right), I}$ be the closed subvariety of $\left(\prod_{i=0}^{t} U_{i}\right) \times$ $\Lambda_{\left(r_{i}\right)}$ consisting of those $\left(u_{i}, A_{i}, B_{i}\right)$ which satisfy the equations

$$
\begin{aligned}
B_{i} u_{i+1} & =u_{i}\left(\text { equation in } U_{i}\right), \quad \text { for all } i \in I, \text { and } \\
A_{i} u_{i} & =u_{i+1}\left(\text { equation in } U_{i+1}\right), \text { for all } i \in \bar{I} .
\end{aligned}
$$

Since $u_{0}=0$, the second equation implies that $u_{1}=\cdots=u_{k}=0$, where $k$ is the minimal element of $I$; or that $u_{1}=\cdots=u_{t}=0$, in the case that $I=\varnothing$. More generally, the two equations imply that all $u_{i}$ can be determined from those indexed by $i \in(I+1) \backslash I$. 
EXAMPLE 4.7. In the variety $\Lambda_{(1,1,2),\{0,2\}}$ attached to the bipartition $((2,1) ;(1))$, the equations satisfied by $u_{0}, u_{1}, u_{2}$, and $u_{3}$ are $B_{0} u_{1}=u_{0}$, $A_{1} u_{1}=u_{2}$, and $B_{2} u_{3}=u_{2}$. The first of these equations is automatic because $U_{0}=0$. Setting $u=u_{1}$ and $v=u_{3}$, we eliminate $u_{2}$ and get the single equation $A_{1} u=B_{2} v$, recovering Example 1.3.

The action of $\prod_{i=0}^{t} \operatorname{GL}\left(U_{i}\right)$ on $\Lambda_{\left(r_{i}\right)}$ extends to $\Lambda_{\left(r_{i}\right), I}$ in the obvious way:

$$
\left(g_{i}\right) \cdot\left(u_{i}, A_{i}, B_{i}\right)=\left(g_{i} u_{i}, g_{i+1} A_{i} g_{i}^{-1}, g_{i} B_{i} g_{i+1}^{-1}\right) .
$$

The naively expected dimension of $\Lambda_{\left(r_{i}\right), I}$ is given by

$$
\begin{aligned}
d_{\left(r_{i}\right), I} & =d_{\left(r_{i}\right)}+\sum_{i=0}^{t} \operatorname{dim} U_{i}-\sum_{i \in I} \operatorname{dim} U_{i}-\sum_{i \in \bar{I}} \operatorname{dim} U_{i+1} \\
& =d_{\left(r_{i}\right)}+\sum_{i=0}^{t}\left(r_{0}+\cdots+r_{i-1}\right)-\sum_{i \in I}\left(r_{0}+\cdots+r_{i-1}\right)-\sum_{i \in \bar{I}}\left(r_{0}+\cdots+r_{i}\right) \\
& =d_{\left(r_{i}\right)}+\sum_{i \in I} r_{i}=d_{\left(r_{i}\right)}+|\mu| .
\end{aligned}
$$

Recall that the conventions in force imply that $r_{0} \leq r_{1} \leq \cdots \leq r_{t-1}$, and also that whenever $r_{i}=r_{i+1}$ and $i+1 \in I$, we have $i \in I$ as well. Theorem 4.4 is a special case (where $I=\varnothing$ ) of the following conjecture.

COnjeCture 4.8. The variety $\Lambda_{\left(r_{i}\right), I}$ is a normal complete intersection of dimension $d_{\left(r_{i}\right), I}$.

ExAmple 4.9. Suppose that $t=2$ as in Example 4.3. For the four different possible $I$, the equations required of $u_{1} \in U_{1}$ and $u_{2} \in U_{2}$ are as follows:

$$
\begin{aligned}
& I=\emptyset: u_{1}=u_{2}=0, \quad I=\{0\}: A_{1} u_{1}=u_{2}, \\
& I=\{1\}: u_{1}=0, B_{1} u_{2}=0, \quad I=\{0,1\}: B_{1} u_{2}=u_{1} .
\end{aligned}
$$

So $\Lambda_{\left(r_{0}, r_{1}\right), \emptyset} \cong \Lambda_{\left(r_{0}, r_{1}\right)}, \Lambda_{\left(r_{0}, r_{1}\right),\{0\}} \cong U_{1} \times \Lambda_{\left(r_{0}, r_{1}\right)}$, and $\Lambda_{\left(r_{0}, r_{1}\right),\{0,1\}} \cong U_{2} \times$ $\Lambda_{\left(r_{0}, r_{1}\right)}$; in all these cases Conjecture 4.8 is an immediate consequence of Theorem 4.4. If $I=\{1\}$, then by assumption we have $r_{0}<r_{1}$, and $\Lambda_{\left(r_{0}, r_{1}\right),\{1\}}$ may be proved to be irreducible of dimension $d_{\left(r_{0}, r_{1}\right),\{1\}}$ by an argument similar to that in Example 4.3, using the dense open subvariety consisting of triples $\left(u_{2}, A_{1}, B_{1}\right)$, where $\operatorname{dim}\left(\operatorname{im}\left(A_{1}\right)+\mathbb{C} u_{2}\right)=r_{0}+1$. The normality 
of $\Lambda_{\left(r_{0}, r_{1}\right),\{1\}}$ will be proved later, as a special case of Theorem 6.9. (If we allowed $r_{0}$ to equal $r_{1}$, we would find that $\Lambda_{\left(r_{0}, r_{1}\right),\{1\}}$ had two irreducible components.)

The following special case of Conjecture 4.8 is immediate from Kraft and Procesi's result.

Proposition 4.10. If $I=\{0,1, \ldots, s-1\}$ for some $0 \leq s \leq t$, then $\Lambda_{\left(r_{i}\right), I}$ is a normal complete intersection of dimension $d_{\left(r_{i}\right), I}$.

Proof. When $I$ has this special form, the conditions on the $u_{i}$ for $\left(u_{i}, A_{i}\right.$, $\left.B_{i}\right) \in \Lambda_{\left(r_{i}\right), I}$ are equivalent to

$$
u_{i}= \begin{cases}B_{i+1} B_{i+2} \cdots B_{s-1} u_{s} & \text { if } i<s, \\ A_{i-1} A_{i-2} \cdots A_{s} u_{s} & \text { if } i>s .\end{cases}
$$

Hence, $\Lambda_{\left(r_{i}\right), I} \cong U_{s} \times \Lambda_{\left(r_{i}\right)}$. Moreover,

$$
d_{\left(r_{i}\right), I}=d_{\left(r_{i}\right)}+\sum_{i=0}^{s-1} r_{i}=d_{\left(r_{i}\right)}+\operatorname{dim} U_{s} .
$$

So the result follows from Theorem 4.4.

In the next two sections we will make further progress on Conjecture 4.8, culminating in the proof of a different special case in Theorem 6.9.

\subsection{Normality for enhanced nilpotent orbits}

As mentioned in the introduction, Conjecture 4.8 implies Conjecture 1.1 by virtue of the following result.

THEOREM 4.11. If $\Lambda_{\mu ; \nu}=\Lambda_{\left(r_{i}\right), I}$ is a normal variety, then $\overline{\mathcal{O}_{\mu ; \nu}}$ is normal.

To prove Theorem 4.11, it suffices to exhibit $\overline{\mathcal{O}_{\mu ; \nu}}$ as an invariant-theoretic quotient of $\Lambda_{\left(r_{i}\right) ; I}$ by a reductive group, since passage to such a quotient preserves normality. The precise statement, generalizing the $I=\varnothing$ case proved by Kraft and Procesi [KP1, Theorem 3.3], is as follows.

Proposition 4.12. Let $H=\mathrm{GL}\left(U_{0}\right) \times \cdots \times \mathrm{GL}\left(U_{t-1}\right)$. Then the map

$$
\Phi: \Lambda_{\left(r_{i}\right), I} \rightarrow U_{t} \times \mathcal{N}_{U_{t}}:\left(u_{i}, A_{i}, B_{i}\right) \mapsto\left(u_{t}, A_{t-1} B_{t-1}\right)
$$

has image $\overline{\mathcal{O}_{\mu ; \nu}}$ and induces an isomorphism

$$
H \backslash \Lambda_{\left(r_{i}\right), I} \stackrel{\sim}{\rightarrow} \overline{\mathcal{O}_{\mu ; \nu}}
$$


Proof. We proceed by induction on $t$. If $t=1$, then $H$ is the trivial group. If $I=\varnothing$, then $\Lambda_{\left(r_{i}\right), I}$ consists of the single point where $u_{0}=u_{1}=0$ and $A_{0}=B_{0}=0$, and $\mathcal{O}_{\mu ; \nu}=\mathcal{O}_{\varnothing ; 1^{r_{0}}}=\{(0,0)\}$, so the result holds. On the other hand, if $I=\{0\}$, then $\Lambda_{\left(r_{i}\right), I}$ consists of the tuples $\left(u_{0}, u_{1}, A_{0}, B_{0}\right)$ where $u_{0}=0, A_{0}=B_{0}=0$, and $u_{1}$ is arbitrary, and $\overline{\mathcal{O}_{\mu ; \nu}}=\overline{\mathcal{O}_{1^{r_{0}} ; \varnothing}}=U_{1} \times\{0\}$, so the result holds in this case as well.

Now, suppose $t>1$, and let us put

$$
\Lambda^{\prime}=\Lambda_{\left(r_{0}, r_{1}, \ldots, r_{t-2}\right), I \cap\{0, \ldots, t-2\}}
$$

This is an enhanced quiver variety associated to a bipartition $\left(\mu^{\prime} ; \nu^{\prime}\right)$ of total size $r_{0}+\cdots+r_{t-2}$. Let $H^{\prime}=\mathrm{GL}\left(U_{0}\right) \times \cdots \times \mathrm{GL}\left(U_{t-2}\right)$. Then, by assumption, the map

$$
\Phi^{\prime}: \Lambda^{\prime} \rightarrow U_{t-1} \times \mathcal{N}_{U_{t-1}}:\left(u_{i}, A_{i}, B_{i}\right) \mapsto\left(u_{t-1}, A_{t-2} B_{t-2}\right)
$$

has image $\overline{\mathcal{O}_{\mu^{\prime} ; \nu^{\prime}}}$ and induces an isomorphism

$$
H^{\prime} \backslash \backslash \Lambda^{\prime} \stackrel{\sim}{\rightarrow} \overline{\mathcal{O}_{\mu^{\prime} ; \nu^{\prime}}}
$$

Consider the variety

$$
Y= \begin{cases}\left(p_{U_{t-1}}^{U_{t-1}, U_{t}}\right)^{-1}\left(\overline{\mathcal{O}_{\mu^{\prime} ; \nu^{\prime}}}\right) \subset U_{t-1} \times \mathcal{N}_{U_{t-1}, U_{t}} & \text { if } t-1 \in \bar{I}, \\ \left(p_{U_{t-1}, U_{t-1}}^{U_{t}}\right)^{-1}\left(\overline{\mathcal{O}_{\mu^{\prime} ; \nu^{\prime}}}\right) \subset U_{t} \times \mathcal{N}_{U_{t-1}, U_{t}} & \text { if } t-1 \in I\end{cases}
$$

For simplicity, we omit the superscripts on the maps $p_{U_{t-1}}$ and $p_{U_{t}}$ which distinguish between the two cases. By Proposition 3.4, we know in both cases that $p_{U_{t}}$ induces an isomorphism

$$
\mathrm{GL}\left(U_{t-1}\right) \backslash \backslash Y \stackrel{\sim}{\rightarrow} \overline{\mathcal{O}_{\mu ; \nu}}
$$

Let us recall how to describe a point of $\Lambda_{\left(r_{i}\right), I}$. If $t-1 \in I$, then by definition, a point $x$ of $\Lambda_{\left(r_{i}\right), I}$ consists of a point $p(x)=\left(u_{i}, A_{i}, B_{i}\right)$ of $\Lambda^{\prime}$ together with the additional choice of $\left(u_{t}, A_{t-1}, B_{t-1}\right) \in U_{t} \times \mathcal{N}_{U_{t-1}, U_{t}}$ subject to the constraints that $B_{t-1} A_{t-1}=A_{t-2} B_{t-2}$ and $B_{t-1} u_{t}=u_{t-1}$. These constraints say exactly that $\Phi^{\prime}(p(x))=p_{U_{t-1}}\left(u_{t}, A_{t-1}, B_{t-1}\right)$, so we have a canonical identification

$$
\Lambda_{\left(r_{i}\right), I} \cong \Lambda^{\prime} \times_{U_{t-1} \times \mathcal{N}_{U_{t-1}}}\left(U_{t} \times \mathcal{N}_{U_{t-1}, U_{t}}\right)
$$

where the fiber product is taken with respect to $\Phi^{\prime}$ and $p_{U_{t-1}}$. 
We now turn to the case where $t-1 \in \bar{I}$. In this case, a point $x$ of $\Lambda_{\left(r_{i}\right), I}$ consists of $p(x) \in \Lambda^{\prime}$ together with $\left(u_{t}, A_{t-1}, B_{t-1}\right) \in U_{t} \times \mathcal{N}_{U_{t-1}, U_{t}}$, subject to the constraints that $B_{t-1} A_{t-1}=A_{t-2} B_{t-2}$ and $u_{t}=A_{t-1} u_{t-1}$. To relate this variety to $Y$, we must modify this description slightly. Clearly, $\Lambda_{\left(r_{i}\right), I}$ is isomorphic to the subvariety $\hat{\Lambda} \subset \Lambda_{\left(r_{i}\right) I} \times U_{t-1}$ consisting of pairs $\left(x, v_{t-1}\right)$ in which the extra vector $v_{t-1}$ equals the vector $u_{t-1}$ that already occurs as a component of $x$. To rephrase, a point $\hat{x} \in \hat{\Lambda}$ consists of a point $\hat{p}(\hat{x}) \in \Lambda^{\prime}$ together with a triple $\left(v_{t-1}, A_{t-1}, B_{t-1}\right) \in U_{t-1} \times \mathcal{N}_{U_{t-1}, U_{t}}$ and a vector $u_{t} \in$ $U_{t}$, subject to the constraints that $B_{t-1} A_{t-1}=A_{t-2} B_{t-2}, v_{t-1}=u_{t-1}$, and $u_{t}=A_{t-1} v_{t-1}$. Thus, $\hat{\Lambda}$ can be identified with a twofold fiber product

$$
\Lambda^{\prime} \times_{U_{t-1} \times \mathcal{N}_{U_{t-1}}}\left(U_{t-1} \times \mathcal{N}_{U_{t-1}, U_{t}}\right) \times_{U_{t}} U_{t},
$$

with respect to the maps $\Phi^{\prime}, p_{U_{t-1}}, p_{U_{t}}$, and $\mathrm{id}_{U_{t}}$. But now we can clearly drop the last factor in the fiber product: we have

$$
\Lambda_{\left(r_{i}\right), I} \cong \hat{\Lambda} \cong \Lambda^{\prime} \times_{U_{t-1} \times \mathcal{N}_{U_{t-1}}}\left(U_{t-1} \times \mathcal{N}_{U_{t-1}, U_{t}}\right) .
$$

We now observe that in both (4.1) and (4.2), the map $\Phi^{\prime}$ factors through $\overline{\mathcal{O}_{\mu^{\prime} ; \nu^{\prime}}}$. Thus, in both cases, we actually have a diagram as follows, in which both squares are Cartesian:

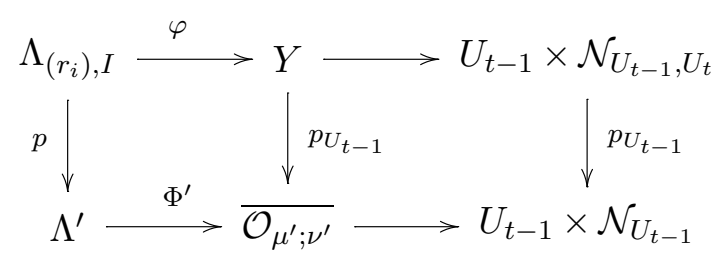

Here, $\varphi: \Lambda_{\left(r_{i}\right), I} \rightarrow Y$ is the map defined by

$$
\varphi(x)= \begin{cases}\left(u_{t-1}, A_{t-1}, B_{t-1}\right) & \text { if } t-1 \in \bar{I}, \\ \left(u_{t}, A_{t-1}, B_{t-1}\right) & \text { if } t-1 \in I .\end{cases}
$$

From the left-hand square in (4.3), we deduce that $\varphi$ induces an isomorphism $H^{\prime} \backslash \backslash \Lambda_{\left(r_{i}\right), I} \stackrel{\sim}{\rightarrow} Y$. Since $\Phi=p_{U_{t}} \circ \varphi$, the result follows.

By Proposition 4.10, we know that $\Lambda_{\left(r_{i}\right), I}$ is normal when $I=\{0,1, \ldots, s-$ $1\}$ for some $s$. This condition is equivalent to requiring that every column of $\nu$ is at least as long as every column of $\mu$. So Theorem 4.11 completes the proof of Theorem 1.2(2). 


\section{§5. The singular locus of $\Lambda_{\left(r_{i}\right), I}$}

We retain all the notation introduced in Section 4 . Let $\Lambda^{\circ} \subset \Lambda_{\left(r_{i}\right), I}$ be the open subset consisting of points $\left(u_{i}, A_{i}, B_{i}\right)$ such that

$$
\begin{cases}\text { either } A_{j} \text { is injective and } u_{j+1} \notin \operatorname{im}\left(A_{j}\right) & \\ \text { or } B_{j} \text { is surjective } & \text { for all } j \in I, \\ \text { either } A_{j} \text { is injective or } B_{j} \text { is surjective } & \text { for all } j \in \bar{I} .\end{cases}
$$

It is easy to see that $\Lambda^{\circ}$ is nonempty.

In this section, we prove that $\Lambda^{\circ}$ is nonsingular, and we use this to reframe Conjecture 4.8 as a dimension calculation.

Define a morphism of affine varieties

$$
\begin{aligned}
& \Psi: \prod_{i=0}^{t} U_{i} \times \prod_{i=0}^{t-1}\left(\operatorname{Hom}\left(U_{i}, U_{i+1}\right) \times \operatorname{Hom}\left(U_{i+1}, U_{i}\right)\right) \\
& \quad \rightarrow \prod_{i \in I} U_{i} \times \prod_{i \in \bar{I}} U_{i+1} \times \prod_{i=1}^{t-1} \operatorname{End}\left(U_{i}\right)
\end{aligned}
$$

by the rule

$$
\Psi\left(u_{i}, A_{i}, B_{i}\right)=\left(B_{i} u_{i+1}-u_{i}, A_{i} u_{i}-u_{i+1}, B_{i} A_{i}-A_{i-1} B_{i-1}\right) .
$$

Then $\Lambda_{\left(r_{i}\right), I}$ is the variety-theoretic zero fiber of $\Psi$. Let $\Psi^{-1}(0)$ denote the scheme-theoretic zero fiber, in other words, the spectrum of the quotient of the free polynomial ring in indeterminates identified with the coordinates of the $u_{i}, A_{i}$, and $B_{i}$ by the ideal generated by the coordinates of the appropriate vectors $B_{i} u_{i+1}-u_{i}$ and $A_{i} u_{i}-u_{i+1}$ and the matrices $B_{i} A_{i}-$ $A_{i-1} B_{i-1}$. A priori, $\Psi^{-1}(0)$ is a possibly nonreduced scheme, whose reduced subscheme is the variety $\Lambda_{\left(r_{i}\right), I}$.

Proposition 5.1. The variety $\Lambda^{\circ}$ is nonsingular of dimension $d_{\left(r_{i}\right), I}$.

Proof. To prove the proposition, it suffices to show that for fixed $\left(u_{i}, A_{i}\right.$, $\left.B_{i}\right) \in \Lambda^{\circ}$, the differential

$$
d \Psi_{\left(u_{i}, A_{i}, B_{i}\right)}: \bigoplus_{i=0}^{t} U_{i} \oplus \bigoplus_{i=0}^{t-1}\left(\operatorname{Hom}\left(U_{i}, U_{i+1}\right) \oplus \operatorname{Hom}\left(U_{i+1}, U_{i}\right)\right)
$$




$$
\rightarrow \bigoplus_{i \in I} U_{i} \oplus \bigoplus_{i \in \bar{I}} U_{i+1} \oplus \bigoplus_{i=1}^{t-1} \operatorname{End}\left(U_{i}\right)
$$

is surjective. This differential maps $\left(u_{i}^{\prime}, A_{i}^{\prime}, B_{i}^{\prime}\right)$ to

$$
\begin{array}{r}
\left(B_{i}^{\prime} u_{i+1}+B_{i} u_{i+1}^{\prime}-u_{i}^{\prime}, A_{i}^{\prime} u_{i}+A_{i} u_{i}^{\prime}-u_{i+1}^{\prime}\right. \\
\left.B_{i}^{\prime} A_{i}+B_{i} A_{i}^{\prime}-A_{i-1}^{\prime} B_{i-1}-A_{i-1} B_{i-1}^{\prime}\right) .
\end{array}
$$

The proof of surjectivity is a slight elaboration of the proof of [KP1, Proposition 3.5]. We introduce filtrations of the domain and codomain:

$$
\begin{aligned}
& 0=X_{t} \subset X_{t-1} \subset \cdots \subset X_{0}=\bigoplus_{i=0}^{t} U_{i} \oplus \bigoplus_{i=0}^{t-1}\left(\operatorname{Hom}\left(U_{i}, U_{i+1}\right) \oplus \operatorname{Hom}\left(U_{i+1}, U_{i}\right)\right), \\
& \text { where } X_{j}=\bigoplus_{i=j+1}^{t} U_{i} \oplus \bigoplus_{i=j}^{t-1}\left(\operatorname{Hom}\left(U_{i}, U_{i+1}\right) \oplus \operatorname{Hom}\left(U_{i+1}, U_{i}\right)\right), \\
& \text { and } 0=Y_{t} \subset Y_{t-1} \subset \cdots \subset Y_{0}=\bigoplus_{i \in I} U_{i} \oplus \bigoplus_{i \in \bar{I}} U_{i+1} \oplus \bigoplus_{i=1}^{t-1} \operatorname{End}\left(U_{i}\right), \\
& \text { where } Y_{j}=\bigoplus_{\substack{i \in I \\
i \geq j}} U_{i} \oplus \bigoplus_{i \in \bar{I}} U_{i+1} \oplus \bigoplus_{i=j}^{t-1} \operatorname{End}\left(U_{i}\right) .
\end{aligned}
$$

It is immediate from the above formula that $d \Psi_{\left(u_{i}, A_{i}, B_{i}\right)}\left(X_{j}\right) \subset Y_{j}$ for all $j$, so it suffices to show that the induced map $\psi_{j}: X_{j} / X_{j+1} \rightarrow Y_{j} / Y_{j+1}$ is surjective for all $0 \leq j \leq t-1$.

If $j \in I$, then $\psi_{j}$ can be identified with the map

$$
\begin{array}{r}
U_{j+1} \oplus \operatorname{Hom}\left(U_{j}, U_{j+1}\right) \oplus \operatorname{Hom}\left(U_{j+1}, U_{j}\right) \rightarrow U_{j} \oplus \operatorname{End}\left(U_{j}\right) \\
\left(u_{j+1}^{\prime}, A_{j}^{\prime}, B_{j}^{\prime}\right) \mapsto\left(B_{j}^{\prime} u_{j+1}+B_{j} u_{j+1}^{\prime}, B_{j}^{\prime} A_{j}+B_{j} A_{j}^{\prime}\right) .
\end{array}
$$

This is surjective because, by the assumption that $\left(u_{i}, A_{i}, B_{i}\right) \in \Lambda^{\circ}$, either $B_{j}$ is surjective (allowing any image to be obtained by varying $u_{j+1}^{\prime}$ and $A_{j}^{\prime}$, with $B_{j}^{\prime}$ set to zero) or the matrix formed by adding $u_{j+1}$ as an extra column to $A_{j}$ has full rank (allowing any image to be obtained by varying $B_{j}^{\prime}$, with $u_{j+1}^{\prime}$ and $A_{j}^{\prime}$ set to zero). 
If $j \in \bar{I}$, then $\psi_{j}$ can be identified with the map

$$
\begin{gathered}
U_{j+1} \oplus \operatorname{Hom}\left(U_{j}, U_{j+1}\right) \oplus \operatorname{Hom}\left(U_{j+1}, U_{j}\right) \rightarrow U_{j+1} \oplus \operatorname{End}\left(U_{j}\right) \\
\left(u_{j+1}^{\prime}, A_{j}^{\prime}, B_{j}^{\prime}\right) \mapsto\left(A_{j}^{\prime} u_{j}-u_{j+1}^{\prime}, B_{j}^{\prime} A_{j}+B_{j} A_{j}^{\prime}\right) .
\end{gathered}
$$

This is surjective because either $B_{j}$ is surjective (allowing any image to be obtained by varying $u_{j+1}^{\prime}$ and $A_{j}^{\prime}$, with $B_{j}^{\prime}$ set to zero) or $A_{j}$ is injective (allowing any image to be obtained by varying $u_{j+1}^{\prime}$ and $B_{j}^{\prime}$, with $A_{j}^{\prime}$ set to zero).

We end this section by showing how to reduce Conjecture 4.8 to the following dimension bound.

Conjecture 5.2. We have $\operatorname{dim}\left(\Lambda_{\left(r_{i}\right), I} \backslash \Lambda^{\circ}\right) \leq d_{\left(r_{i}\right), I}-2$.

THEOREM 5.3. If $\operatorname{dim}\left(\Lambda_{\left(r_{i}\right), I} \backslash \Lambda^{\circ}\right) \leq d_{\left(r_{i}\right), I}-2$, then $\Psi^{-1}(0) \cong \Lambda_{\left(r_{i}\right), I}$, and this variety is a normal complete intersection of dimension $d_{\left(r_{i}\right), I}$.

Proof. If we let $f(i)$ be the positive integer $t+1+i-2|I \cap\{0, \ldots, i-1\}|$ for $0 \leq i \leq t$, then for all $\alpha \in \mathbb{C}^{\times}$,

$$
\begin{aligned}
& \Psi\left(\alpha^{f(i)} u_{i}, \alpha A_{i}, \alpha B_{i}\right) \\
& \quad=\left(\alpha^{f(i)}\left(B_{i} u_{i+1}-u_{i}\right), \alpha^{f(i+1)}\left(A_{i} u_{i}-u_{i+1}\right), \alpha^{2}\left(B_{i} A_{i}-A_{i-1} B_{i-1}\right)\right) .
\end{aligned}
$$

Hence, $\Psi^{-1}(0)$ is connected, because it is a cone over a subscheme of weighted projective space (with all weights positive).

From Proposition 5.1 and the assumption on $\operatorname{dim}\left(\Lambda_{\left(r_{i}\right), I} \backslash \Lambda^{\circ}\right)$, we see that the scheme $\Psi^{-1}(0)$ is a connected complete intersection which is regular in codimension 1. By Serre's criterion, $\Psi^{-1}(0)$ is reduced, irreducible, and normal; it therefore coincides with the variety $\Lambda_{\left(r_{i}\right), I}$, as desired.

\section{$\S 6$. Stratifications and dimension estimates}

In this section, we endow $\Lambda_{\left(r_{i}\right), I}$ with a stratification, and we estimate the dimension of each stratum. This dimension estimate enables us to reduce Conjecture 5.2 to a purely combinatorial statement about sequences of signed quasi-bipartitions.

For $0 \leq j \leq t-1$, let us define

$$
\hat{\mathcal{N}}_{j}=\left\{\begin{array}{ll}
U_{j} \times \mathcal{N}_{U_{j}, U_{j+1}} & \text { if } j \in \bar{I}, \\
U_{j+1} \times \mathcal{N}_{U_{j}, U_{j+1}} & \text { if } j \in I
\end{array} \quad\right. \text { and }
$$




$$
\hat{\mathcal{Q}}_{j}= \begin{cases}\mathcal{S} \mathcal{Q}_{\operatorname{dim} U_{j}, \operatorname{dim} U_{j+1}} & \text { if } j \in \bar{I} \\ \mathcal{S} \mathcal{Q}_{\operatorname{dim} U_{j+1}, \operatorname{dim} U_{j}} & \text { if } j \in I\end{cases}
$$

Recall from Lemma 2.7 that the $\left(\mathrm{GL}\left(U_{j}\right) \times \mathrm{GL}\left(U_{j+1}\right)\right)$-orbits in $\hat{\mathcal{N}}_{j}$ are parameterized by the set of signed quasi-bipartitions $\hat{\mathcal{Q}}_{j}$. There is an obvious map $h_{j}: \Lambda_{\left(r_{i}\right), I} \rightarrow \hat{\mathcal{N}}_{j}$ which forgets all but the relevant vector and nilpotent pair, and we define a map

$$
\Theta: \Lambda_{\left(r_{i}\right), I} \rightarrow \prod_{j=0}^{t-1} \hat{\mathcal{Q}}_{j}
$$

by associating to each point $\left(u_{i}, A_{i}, B_{i}\right) \in \Lambda_{\left(r_{i}\right), I}$ the sequence of signed quasi-bipartitions labeling the $\left(\mathrm{GL}\left(U_{j}\right) \times \mathrm{GL}\left(U_{j+1}\right)\right)$-orbit of $h_{j}\left(u_{i}, A_{i}, B_{i}\right)$ for each $j$. Let $\Xi$ be the image of this map. We endow $\Lambda_{\left(r_{i}\right), I}$ with a stratification indexed by $\Xi$ by taking the strata to be the fibers of the map above:

$$
\Lambda_{\left(r_{i}\right), I}^{\xi}=\Theta^{-1}(\xi) \text { for any } \xi=\left(\xi_{j}\right) \in \Xi \text {. }
$$

It is clear that each $\Lambda_{\left(r_{i}\right), I}^{\xi}$ is a locally closed subvariety of $\Lambda_{\left(r_{i}\right), I}$ whose boundary is a union of smaller such strata. The strata are clearly preserved by the action of $\prod_{i=0}^{t} \mathrm{GL}\left(U_{i}\right)$.

Each $\xi=\left(\xi_{j}\right) \in \Xi$ determines a sequence of bipartitions $\left(\rho_{\xi}^{(j)} ; \sigma_{\xi}^{(j)}\right)$ for $1 \leq j \leq t$, by the following condition:

$$
\left(u_{j}, A_{j-1} B_{j-1}\right) \in \mathcal{O}_{\rho_{\xi}^{(j)} ; \sigma_{\xi}^{(j)}} \subset U_{j} \times \mathcal{N}_{U_{j}} \quad \text { for all }\left(u_{i}, A_{i}, B_{i}\right) \in \Lambda_{\left(r_{i}\right), I}^{\xi} .
$$

We also set $\left(\rho_{\xi}^{(0)} ; \sigma_{\xi}^{(0)}\right)=(\varnothing ; \varnothing)$. Thus, for each $0 \leq j \leq t-1,\left(\rho_{\xi}^{(j)} ; \sigma_{\xi}^{(j)}\right)$ and $\left(\rho_{\xi}^{(j+1)} ; \sigma_{\xi}^{(j+1)}\right)$ are the bipartitions subordinate to the signed quasibipartition $\xi_{j}$, with + corresponding to $U_{j}$ if $j \in \bar{I}$ and to $U_{j+1}$ if $j \in I$. The condition for $\xi$ to lie in $\Xi$ is exactly that the subordinate bipartitions of adjacent $\xi_{j}$ match up in this way.

Example 6.1. Continue with Example 4.7. A stratum of $\Lambda_{(1,1,2),\{0,2\}}$ is indexed by a sequence of signed quasi-bipartitions $\xi=\left(\xi_{0}, \xi_{1}, \xi_{2}\right)$, where $\xi_{0} \in \mathcal{S} \mathcal{Q}_{1,0}, \xi_{1} \in \mathcal{S} \mathcal{Q}_{1,2}$, and $\xi_{2} \in \mathcal{S} \mathcal{Q}_{4,2}$. The compatibility conditions these must satisfy are that the + subordinate bipartition of $\xi_{0}$ equals that of $\xi_{1}$ (this is $\left.\left(\rho_{\xi}^{(1)} ; \sigma_{\xi}^{(1)}\right)\right)$ and that the - subordinate bipartition of $\xi_{1}$ equals that 
of $\xi_{2}$ (this is $\left.\left(\rho_{\xi}^{(2)} ; \sigma_{\xi}^{(2)}\right)\right)$. For instance, the zero stratum corresponds to

$$
\xi_{0}=\boxplus \quad \xi_{1}=\mathrm{E} \quad \xi_{2}=
$$

and the unique stratum of maximal dimension (see Conjecture 6.5(2), which is proved in this case) corresponds to

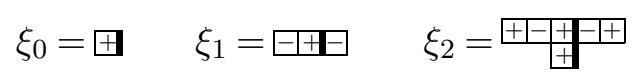

A third stratum is described in Example 6.8.

Proposition 6.2. Each $\Lambda_{\left(r_{i}\right), I}^{\xi}$ is a smooth variety. Moreover, we have

(6.1) $\operatorname{dim} \Lambda_{\left(r_{i}\right), I}^{\xi} \leq d_{\left(r_{i}\right)}+n(\lambda)-n\left(\rho_{\xi}^{(t)}+\sigma_{\xi}^{(t)}\right)-\sum_{\substack{i \in I \\ i-1 \in \bar{I}}}\left|\rho_{\xi}^{(i)}\right|+\sum_{\substack{i \in \bar{I} \cup\{t\} \\ i-1 \in I}}\left|\rho_{\xi}^{(i)}\right|$.

Proof. For $0 \leq j \leq t-1$, let $\mathcal{C}_{j}=h_{j}\left(\Lambda_{\left(r_{i}\right), I}^{\xi}\right)$. This variety is, by definition, a single $\left(\mathrm{GL}\left(U_{j}\right) \times \operatorname{GL}\left(U_{j+1}\right)\right)$-orbit in $\hat{\mathcal{N}}_{j}$. For $0 \leq j \leq t$, let $\mathcal{O}_{j}$ be the $\operatorname{GL}\left(U_{j}\right)$-orbit in $U_{j} \times \mathcal{N}_{U_{j}}$ labeled by the bipartition $\left(\rho_{\xi}^{(j)} ; \sigma_{\xi}^{(j)}\right)$. Finally, let $\mathcal{P}_{j}=\bar{\pi}^{U_{j}}\left(\mathcal{O}_{j}\right)$; this is the $\operatorname{GL}\left(U_{j}\right)$-orbit in $\mathcal{N}_{U_{j}}$ labeled by $\rho_{\xi}^{(j)}+\sigma_{\xi}^{(j)}$.

It is easy to see from the definition that $\Lambda_{\left(r_{i}\right), I}^{\xi}$ is isomorphic to the fiber product

$$
\mathcal{C}_{0} \times{ }_{\mathcal{O}_{1}} \mathcal{C}_{1} \times \mathcal{O}_{2} \cdots \times{ }_{\mathcal{O}_{t-1}} \mathcal{C}_{t-1}
$$

Since the varieties $\mathcal{C}_{i}$ and the morphisms $\mathcal{C}_{i} \rightarrow \mathcal{O}_{i}$ and $\mathcal{C}_{i} \rightarrow \mathcal{O}_{i-1}$ are all smooth, it follows that $\Lambda_{\left(r_{i}\right), I}^{\xi}$ is smooth and that its dimension is given by

$$
\operatorname{dim} \Lambda_{\left(r_{i}\right), I}^{\xi}=\sum_{i=0}^{t-1} \operatorname{dim} \mathcal{C}_{i}-\sum_{i=1}^{t-1} \operatorname{dim} \mathcal{O}_{i}
$$

Since $\operatorname{dim} \mathcal{O}_{0}=0$, there is no harm in changing this formula to

$$
\operatorname{dim} \Lambda_{\left(r_{i}\right), I}^{\xi}=\sum_{i=0}^{t-1}\left(\operatorname{dim} \mathcal{C}_{i}-\operatorname{dim} \mathcal{O}_{i}\right)
$$

From Lemma 2.10, we have that

$$
\operatorname{dim} \mathcal{C}_{i}-\operatorname{dim} \mathcal{O}_{i} \leq \frac{1}{2}\left(\operatorname{dim} \mathcal{P}_{i+1}-\operatorname{dim} \mathcal{P}_{i}\right)+\left(\operatorname{dim} U_{i}\right)\left(\operatorname{dim} U_{i+1}\right)
$$




$$
+ \begin{cases}0 & \text { if } i \in \bar{I} \\ \left|\rho_{\xi}^{(i+1)}\right|-\left|\rho_{\xi}^{(i)}\right| & \text { if } i \in I .\end{cases}
$$

Summing up over $i \in\{0,1, \ldots, t-1\}$, we find that

$$
\operatorname{dim} \Lambda_{\left(r_{i}\right), I}^{\xi} \leq \frac{1}{2} \operatorname{dim} \mathcal{P}_{t}+\sum_{i=0}^{t-1}\left(\operatorname{dim} U_{i}\right)\left(\operatorname{dim} U_{i+1}\right)+\sum_{\substack{i \in \bar{I} \cup\{t\} \\ i-1 \in I}}\left|\rho_{\xi}^{(i)}\right|-\sum_{\substack{i \in I \\ i-1 \in \bar{I}}}\left|\rho_{\xi}^{(i)}\right|
$$

The result then follows from the dimension formula (2.5), in the form

$$
\frac{1}{2} \operatorname{dim} \mathcal{P}_{t}=\frac{1}{2}\left(\operatorname{dim} U_{t}\right)^{2}-\frac{1}{2} \operatorname{dim} U_{t}-n\left(\rho_{\xi}^{(t)}+\sigma_{\xi}^{(t)}\right)
$$

and the following calculation:

$$
\begin{aligned}
\frac{1}{2}( & \left.\operatorname{dim} U_{t}\right)^{2}-\frac{1}{2} \operatorname{dim} U_{t}+\sum_{i=0}^{t-1}\left(\operatorname{dim} U_{i}\right)\left(\operatorname{dim} U_{i+1}\right) \\
= & \frac{1}{2}\left(r_{0}+\cdots+r_{t-1}\right)^{2}-\frac{1}{2}\left(r_{0}+\cdots+r_{t-1}\right) \\
& \quad+\sum_{i=0}^{t-1}\left(r_{0}+\cdots+r_{i-1}\right)\left(r_{0}+\cdots+r_{i}\right) \\
= & \frac{1}{2} \sum_{i=0}^{t-1}\left(r_{i}^{2}-r_{i}\right)+\sum_{0 \leq i<j \leq t-1} r_{i} r_{j}+\sum_{i=0}^{t-1}\left(r_{0}+\cdots+r_{i-1}\right)^{2}+\sum_{0 \leq i<j \leq t-1} r_{i} r_{j} \\
= & \sum_{i=0}^{t-1}\left(\begin{array}{c}
r_{i} \\
2
\end{array}\right)+d_{\left(r_{i}\right)} \\
= & d_{\left(r_{i}\right)}+n(\lambda) .
\end{aligned}
$$

Note that we could have derived an exact formula for $\operatorname{dim} \Lambda_{\left(r_{i}\right), I}^{\xi}$ by the same argument if we had used the formula [J, Corollary 5.9] for $\operatorname{dim} \mathcal{C}_{i}$ rather than Lemma 2.10. However, this would have introduced even more combinatorial complexity, and we do not need it for Theorem 6.9 below.

Recall from Proposition 4.12 that $\Phi\left(\Lambda_{\left(r_{i}\right), I}\right)=\overline{\mathcal{O}_{\mu ; \nu}}$, where $\Phi:\left(u_{i}, A_{i}\right.$, $\left.B_{i}\right) \mapsto\left(u_{t}, A_{t-1} B_{t-1}\right)$. In particular, we have

$$
\mathcal{O}_{\rho_{\xi}^{(t)} ; \sigma_{\xi}^{(t)}}=\Phi\left(\Lambda_{\left(r_{i}\right), I}^{\xi}\right) \subset \overline{\mathcal{O}_{\mu ; \nu}} \quad \text { and hence } \quad \mathcal{O}_{\rho_{\xi}^{(t)}+\sigma_{\xi}^{(t)}} \subset \overline{\mathcal{O}_{\lambda}} .
$$


By the dimension formula (2.5), this implies that $n\left(\rho_{\xi}^{(t)}+\sigma_{\xi}^{(t)}\right) \geq n(\lambda)$. We have thus proved the following additional inequality.

Corollary 6.3. In the setting of Proposition 6.2, we have

$$
\operatorname{dim} \Lambda_{\left(r_{i}\right), I}^{\xi} \leq d_{\left(r_{i}\right)}-\sum_{\substack{i \in I \\ i-1 \in \bar{I}}}\left|\rho_{\xi}^{(i)}\right|+\sum_{\substack{i \in \bar{I} \cup\{t\} \\ i-1 \in I}}\left|\rho_{\xi}^{(i)}\right| .
$$

The Kraft-Procesi stratification of $\Lambda_{\left(r_{i}\right)}$ is the $I=\varnothing$ special case of the stratification defined above. In this case, the last two terms in Proposition 6.2 and Corollary 6.3 vanish, and those results become dimension bounds obtained in [KP1, Section 5]. In fact, Kraft and Procesi proved the following more precise result.

TheOREm 6.4 ([KP1, Section 5]). Suppose that $I=\varnothing$.

(1) For any $\xi, \operatorname{dim} \Lambda_{\left(r_{i}\right)}^{\xi} \leq d_{\left(r_{i}\right)}$.

(2) Equality holds in (1) for a unique $\xi$ : the corresponding stratum consists of those $\left(A_{i}, B_{i}\right)$ such that, for all $i, A_{i}$ is injective and $B_{i}$ is surjective.

(3) If $\left(A_{i}, B_{i}\right)$ belongs to a stratum $\Lambda_{\left(r_{i}\right)}^{\xi}$ of dimension $d_{\left(r_{i}\right)}-1$, then for each $i$, either $A_{i}$ is injective or $B_{i}$ is surjective.

Motivated by this result, we formulate the following conjecture, which would clearly imply Conjecture 5.2 and hence Conjecture 4.8.

Conjecture 6.5. We have the following.

(1) For any $\xi \in \Xi, \operatorname{dim} \Lambda_{\left(r_{i}\right), I}^{\xi} \leq d_{\left(r_{i}\right), I}$.

(2) Equality holds in (1) for a unique $\xi$ : the corresponding stratum consists of those $\left(u_{i}, A_{i}, B_{i}\right)$ such that $A_{i}$ is injective and $B_{i}$ is surjective for all $i$, and $u_{i+1} \notin \operatorname{im}\left(A_{i}\right)$ for all $i \in I$.

(3) If $\operatorname{dim} \Lambda_{\left(r_{i}\right), I}^{\xi}=d_{\left(r_{i}\right), I}-1$, then $\Lambda_{\left(r_{i}\right), I}^{\xi} \subset \Lambda^{\circ}$.

This formulation of the problem lends itself to purely combinatorial calculations. The set of sequences of signed quasi-bipartitions $\Xi$ has a purely combinatorial description; the dimension upper bound in Proposition 6.2 and the exact formula which we have omitted are combinatorial; and the conditions in Conjecture 6.5(2),(3) admit the following combinatorial descriptions.

Lemma 6.6. Let $\xi=\left(\xi_{j}\right) \in \Xi$, and let $\left(u_{i}, A_{i}, B_{i}\right) \in \Lambda_{\left(r_{i}\right), I}^{\xi}$. 
(1) Consequently, $\left(u_{i}, A_{i}, B_{i}\right)$ satisfies the condition of Conjecture 6.5(2) if and only if, in the signed quasi-bipartitions $\xi_{j}$ for all $j \in \bar{I}$, every row begins and ends with a-box,

and in the signed quasi-bipartitions $\xi_{j}$ for all $j \in I$, $\left\{\begin{array}{c}\text { every row begins and ends with } a+\text { box, and there is a box } \\ \text { immediately left of the wall which is at the end of a row. }\end{array}\right.$

(2) $\left(u_{i}, A_{i}, B_{i}\right) \in \Lambda^{\circ}$ if and only if, in the signed quasi-bipartitions $\xi_{j}$ for all $j \in \bar{I}$,

$$
\left\{\begin{array}{l}
\text { either every row begins with } a-b o x, \\
\text { or every row ends with } a-b o x
\end{array}\right.
$$

and in the signed quasi-bipartitions $\xi_{j}$ for all $j \in I$, $\left\{\begin{array}{l}\text { either every row begins with } a+\text { box and there is a box } \\ \quad \text { immediately left of the wall which is at the end of a row, } \\ \text { or every row ends with } a+b o x .\end{array}\right.$

Proof. This is a straightforward translation of the definitions, using the basis interpretation of the signed quasi-bipartition diagram given in Lemma 2.7.

The authors have implemented a computer program to test Conjecture 6.5 (and therefore all the other conjectures in the paper) and have found that it holds for all cases with $n \leq 6$, with part (1) verified up to $n=9$. This completes the proof of Theorem 1.2(1).

REMARK 6.7. Attempts to prove Conjecture 6.5 have revealed that not all properties which hold in [KP1] have obvious enhanced analogues. For example, in the Kraft-Procesi situation one has

$$
\operatorname{codim}_{\Lambda_{\left(r_{i}\right)}} \Lambda_{\left(r_{i}\right)}^{\xi} \geq \frac{1}{2} \operatorname{codim}_{\overline{\mathcal{O}_{\lambda}}} \Phi\left(\Lambda_{\left(r_{i}\right)}^{\xi}\right)
$$

This is one way of stating the $I=\varnothing$ case of Proposition 6.2; the analogue for other classical groups is [KP2, Lemma 5.4]. However, the obvious enhanced analogue of this inequality, namely,

$$
\operatorname{codim}_{\Lambda_{\left(r_{i}\right), I}} \Lambda_{\left(r_{i}\right), I}^{\xi} \geq \frac{1}{2} \operatorname{codim}_{\overline{\mathcal{O}_{\mu ; \nu}}} \Phi\left(\Lambda_{\left(r_{i}\right), I}^{\xi}\right),
$$

is false in general. 
ExAmPLE 6.8. Continue with Example 6.1. There is a stratum $\Lambda_{(1,1,2),\{0,2\}}^{\xi}$ consisting of all tuples $\left(u_{i}, A_{i}, B_{i}\right)$ such that $u_{1} \neq 0, A_{1}=0, B_{1} \neq 0, A_{2}$ is injective, and $B_{2}$ is surjective. The corresponding signed quasi-bipartitions are as follows:

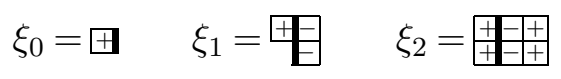

This stratum has codimension 1 in $\Lambda_{(1,1,2),\{0,2\}}$ and belongs to $\Lambda^{\circ}$ in accordance with Conjecture 6.5(3). However, $\Phi\left(\Lambda_{(1,1,2),\{0,2\}}^{\xi}\right)=\mathcal{O}_{(1,1) ;(1,1)}$ has codimension 3 in $\overline{\mathcal{O}_{(2,1) ;(1)}}$, in violation of $(6.5)$.

We now prove our conjectures in the special case which is "opposite" to the one handled in Proposition 4.10.

THEOREM 6.9. If $I=\{s, s+1, \ldots, t-1\}$ for some $1 \leq s \leq t-1$, then Conjecture 6.5 holds.

Proof. First of all, note that the assumption that $I=\{s, s+1, \ldots, t-1\}$ for some $1 \leq s \leq t-1$ is equivalent to saying that every column of $\mu$ is strictly longer than every column of $\nu$ (and both $\mu$ and $\nu$ are nonempty).

We prove the three parts of Conjecture 6.5 in turn. Since $\bar{I}=\{0,1, \ldots, s-$ $1\}$, we have $u_{0}=u_{1}=\cdots=u_{s}=0$ for all $\left(u_{i}, A_{i}, B_{i}\right) \in \Lambda_{\left(r_{i}\right), I}$. Therefore, for any $\xi \in \Xi$, we have $\rho_{\xi}^{(0)}=\rho_{\xi}^{(1)}=\cdots=\rho_{\xi}^{(s)}=\varnothing$. The inequality in Proposition 6.2 reduces to

$$
\operatorname{dim} \Lambda_{\left(r_{i}\right), I}^{\xi} \leq d_{\left(r_{i}\right)}+n(\lambda)-n\left(\rho_{\xi}^{(t)}+\sigma_{\xi}^{(t)}\right)+\left|\rho_{\xi}^{(t)}\right| .
$$

Recall from $(6.3)$ that $\mathcal{O}_{\rho_{\xi}^{(t)} ; \sigma_{\xi}^{(t)}} \subset \overline{\mathcal{O}_{\mu ; \nu}}$. It then follows from Lemma 2.8 that

$$
d_{\left(r_{i}\right)}+n(\lambda)-n\left(\rho_{\xi}^{(t)}+\sigma_{\xi}^{(t)}\right)+\left|\rho_{\xi}^{(t)}\right| \leq d_{\left(r_{i}\right)}+|\mu|=d_{\left(r_{i}\right), I}
$$

Combining (6.6) and (6.7), we deduce Conjecture 6.5(1).

To prove Conjecture 6.5(2), suppose that $\xi \in \Xi$ is such that $\operatorname{dim} \Lambda_{\left(r_{i}\right), I}^{\xi}=$ $d_{\left(r_{i}\right), I}$. Then equality must hold in (6.7), our application of Lemma 2.8, which implies (see Remark 2.9) that $\left(\rho_{\xi}^{(t)} ; \sigma_{\xi}^{(t)}\right)$ is obtained from $(\mu ; \nu)$ by a sequence of type-(4) moves. However, the assumption on the column lengths of $\mu$ and $\nu$ makes a type-(4) move from $(\mu ; \nu)$ impossible, and we conclude that $\left(\rho_{\xi}^{(t)} ; \sigma_{\xi}^{(t)}\right)=(\mu ; \nu)$. In particular, the number of rows of $\xi_{t-1}$ containing $\mathrm{a}+$ box is $\ell(\mu+\nu)=\ell(\mu)=r_{t-1}$. But $r_{t-1}=\operatorname{dim} U_{t}-\operatorname{dim} U_{t-1}$ is also the difference between the number of + boxes and the number of - boxes. 
Hence, $\xi_{t-1}$ must be the signed quasi-bipartition obtained by labeling every box of the diagram of $(\mu ; \nu)$ as + and then inserting a - box between any two adjacent + boxes in the same row. (There is no ambiguity about the position of - boxes adjacent to the wall, since by definition every box immediately left of the wall must be + .) From this, one deduces that $\left(\rho_{\xi}^{(t-1)} ; \sigma_{\xi}^{(t-1)}\right)=$ $\left(\mu^{\prime} ; \nu\right)$, where $\mu^{\prime}$ is obtained from $\mu$ by deleting the longest column. See the top half of Figure 2 for an example of a triple $(\mu ; \nu), \xi_{t-1},\left(\mu^{\prime} ; \nu\right)$ of this form (where $\xi_{t-1}$ is the top signed quasi-bipartition).

Repeating this argument, we obtain for all $j \geq s$ that $\sigma_{\xi}^{(j)}=\nu$ and that $\rho_{\xi}^{(j)}$ is obtained from $\mu$ by deleting the $t-j$ longest columns. Moreover, for all $j \geq s, \xi_{j}$ is uniquely determined: it must be the signed quasi-bipartition obtained by labeling every box of the diagram of $\left(\rho_{\xi}^{(j+1)} ; \sigma_{\xi}^{(j+1)}\right)$ as + and

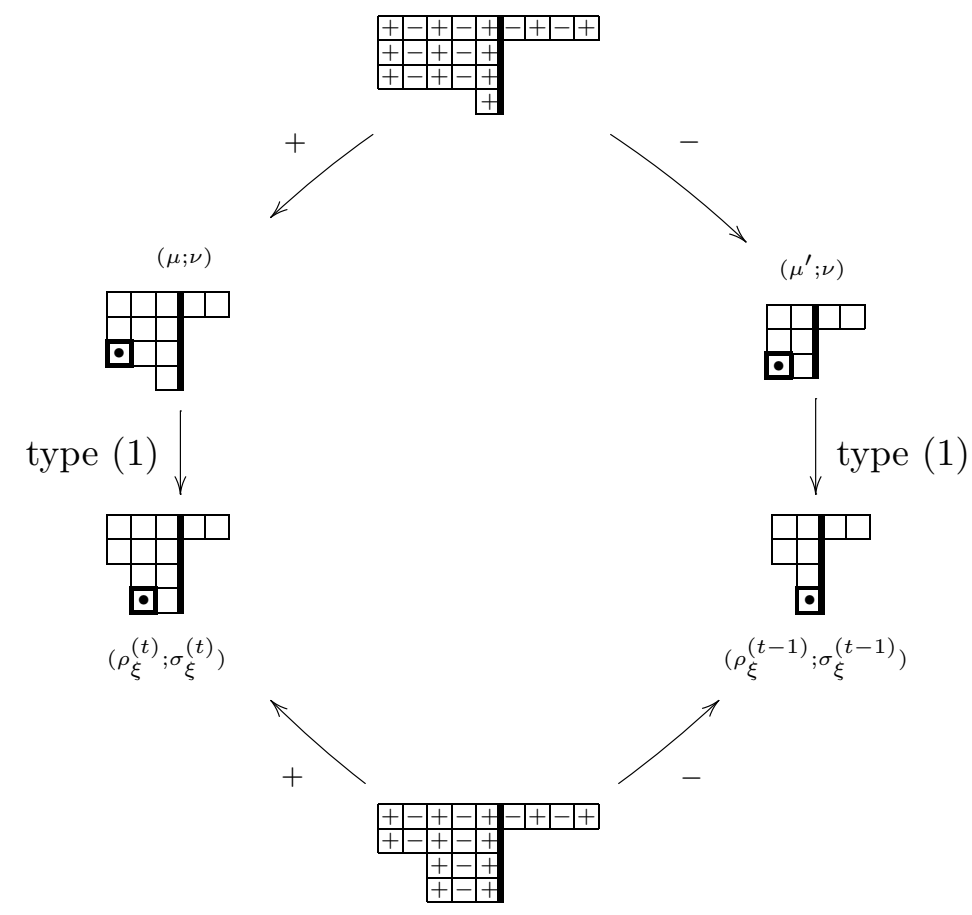

Figure 2: Simultaneous degeneration of subordinate bipartitions 
then inserting - boxes as before. In particular, every row begins and ends with + , and the column of + boxes immediately left of the wall is longer than the column of - boxes immediately right of the wall, as required by Lemma 6.6(1). The corresponding statements for $j<s$ follow in exactly the same way, where now, because $\rho_{\xi}^{(j)}=\varnothing$ for $j \leq s$, we have reverted to the unenhanced case as in Kraft and Procesi's proof of Theorem 6.4(2): one finds that $\sigma_{\xi}^{(j)}$ is obtained from $\nu$ by deleting the $s-j$ longest columns, and $\xi_{j}$ is obtained by labeling every box of $\sigma_{\xi}^{(j+1)}$ as - and then inserting + boxes. (There are, of course, no boxes to the left of the wall.) In particular, every row begins and ends with -, as required by Lemma 6.6(1). As in [KP1, Section 5.4], it is easy to see that this is the only sequence of compatible signed quasi-bipartitions which satisfies the properties required by Lemma 6.6(1), so this stratum does have the description claimed in Conjecture $6.5(2)$. In view of that description, it follows immediately from Theorem $6.4(2)$ that this stratum indeed has dimension $d_{\left(r_{i}\right), I}$. This completes the proof of Conjecture 6.5(2); in fact, we now know the extra information that the only stratum for which equality holds in (6.7) is the stratum we have just described.

To prove Conjecture 6.5(3), let $\xi \in \Xi$ be such that $\operatorname{dim} \Lambda_{\left(r_{i}\right), I}^{\xi}=d_{\left(r_{i}\right), I}-1$. Then equality cannot hold in (6.7), so it must be that equality holds in (6.6) and fails by exactly 1 in (6.7). We aim to prove by induction on $t$ that this implies the conditions required by Lemma 6.6(2).

Let $\xi^{\prime}=\left(\xi_{0}, \ldots, \xi_{t-2}\right)$, let $\left(r_{i}^{\prime}\right)=\left(r_{0}, \ldots, r_{t-2}\right)$, and let $I^{\prime}=\{s, s+1, \ldots$, $t-2\}$. (Note that $I^{\prime}$ is empty if $s=t-1$.) The pair $\left(\left(r_{i}^{\prime}\right), I^{\prime}\right)$ corresponds to the bipartition $\left(\mu^{\prime} ; \nu\right)$, where $\mu^{\prime}$, as above, is obtained by deleting the longest column of $\mu$. From the proof of Proposition 6.2 it is clear that equality in (6.6) forces the corresponding equality:

$$
\operatorname{dim} \Lambda_{\left(r_{i}^{\prime}\right), I^{\prime}}^{\xi^{\prime}}=d_{\left(r_{i}^{\prime}\right)}+n\left(\mu^{\prime}+\nu\right)-n\left(\rho_{\xi}^{(t-1)}+\sigma_{\xi}^{(t-1)}\right)+\left|\rho_{\xi}^{(t-1)}\right| .
$$

So if we can show that equality fails by 1 in the analogue of (6.7), that is, that

$$
d_{\left(r_{i}^{\prime}\right)}+n\left(\mu^{\prime}+\nu\right)-n\left(\rho_{\xi}^{(t-1)}+\sigma_{\xi}^{(t-1)}\right)+\left|\rho_{\xi}^{(t-1)}\right|=d_{\left(r_{i}^{\prime}\right)}+\left|\mu^{\prime}\right|-1
$$

then we will know by the induction hypothesis (or, in the case $s=t-1$, by Theorem 6.4(3)) that the conditions in Lemma 6.6(2) hold for all $j \leq t-2$. Alternatively, if we can show that equality holds in the analogue of (6.7), 
that is, that

$$
d_{\left(r_{i}^{\prime}\right)}+n\left(\mu^{\prime}+\nu\right)-n\left(\rho_{\xi}^{(t-1)}+\sigma_{\xi}^{(t-1)}\right)+\left|\rho_{\xi}^{(t-1)}\right|=d_{\left(r_{i}^{\prime}\right)}+\left|\mu^{\prime}\right|,
$$

then we will know by the above proof of Conjecture 6.5(2) (or, in the case $s=t-1$, by Theorem 6.4(2)) that the conditions in Lemma 6.6(1) hold for all $j \leq t-2$; clearly, these are even stronger than those in Lemma 6.6(2). Assuming either of these eventualities, if we can also show that the condition in Lemma 6.6(2) holds for $j=t-1$, then we will have completed the induction step.

Now by Remark 2.9 and the assumption on column lengths of $\mu$ and $\nu$, saying that equality fails by exactly 1 in (6.7) is equivalent to saying that $\left(\rho_{\xi}^{(t)} ; \sigma_{\xi}^{(t)}\right)$ is obtained from $(\mu ; \nu)$ by one of the following moves:

- a type-(1) move in which the box moves down a single row,

- a type-(2) move in which the box moves down a single row, or

- a type-(3) move in which a single box in row $\ell(\nu)+1$ moves from the bottom of a column of $\mu$ (necessarily of minimal length) to the bottom of a column of $\nu$ (necessarily of maximal length),

possibly followed, in the type-(3) case, by at most two type-(4) moves which have now become possible (because the type-(3) move has disrupted the property that all columns of $\mu$ are strictly longer than all columns of $\nu)$. We now have various cases to consider. Recall that $\ell(\mu)=r_{t-1}$, so $\ell\left(\rho_{\xi}^{(t)}+\sigma_{\xi}^{(t)}\right)$ is either $r_{t-1}$ or $r_{t-1}+1$.

Case 1: $\ell\left(\rho_{\xi}^{(t)}\right)=\ell\left(\rho_{\xi}^{(t)}+\sigma_{\xi}^{(t)}\right)=r_{t-1}$. This implies that the number of rows of $\xi_{t-1}$ containing a + box equals $r_{t-1}$, which as above forces $\xi_{t-1}$ to be the signed quasi-bipartition obtained by labeling every box of the diagram of $\left(\rho_{\xi}^{(t)} ; \sigma_{\xi}^{(t)}\right)$ as + and then inserting - boxes between adjacent + boxes. Since every row ends with a + , the condition in Lemma 6.6(2) holds for $j=t-1$. Moreover, we see that $\sigma_{\xi}^{(t-1)}=\sigma_{\xi}^{(t)}$ and that $\rho_{\xi}^{(t-1)}$ is obtained from $\rho_{\xi}^{(t)}$ by deleting the longest column. Hence, $\left(\rho_{\xi}^{(t-1)} ; \sigma_{\xi}^{(t-1)}\right)$ is obtained from $\left(\mu^{\prime} ; \nu\right)$ by the "same" move (or sequence of moves) which produced $\left(\rho_{\xi}^{(t)} ; \sigma_{\xi}^{(t)}\right)$ from $(\mu ; \nu)$ (for which the possibilities were described above). This implies (6.9), finishing this case. See Figure 2 for an example (where $\xi_{t-1}$ is the bottom signed quasi-bipartition).

Case 2: $\ell\left(\rho_{\xi}^{(t)}\right)<\ell\left(\rho_{\xi}^{(t)}+\sigma_{\xi}^{(t)}\right)=r_{t-1}$. This case occurs if and only if $\mu$ has a single column (so $s=t-1), \ell(\nu)=r_{t-1}-1$, and to form $\left(\rho_{\xi}^{(t)} ; \sigma_{\xi}^{(t)}\right)$ we make a type-(3) move. It is still true that the number of rows of $\xi_{t-1}$ containing 
a + box equals $r_{t-1}$, so as in Case $1, \xi_{t-1}$ is the signed quasi-bipartition obtained by labeling every box of the diagram of $\left(\rho_{\xi}^{(t)} ; \sigma_{\xi}^{(t)}\right)$ as + and then inserting - boxes between adjacent + boxes. Once again, every row ends with a + . Since $\rho_{\xi}^{(t-1)}$ is empty and $\sigma_{\xi}^{(t-1)}=\nu,(6.10)$ holds, finishing this case.

Case 3: $\ell\left(\rho_{\xi}^{(t)}+\sigma_{\xi}^{(t)}\right)=r_{t-1}+1$ and $\left|\rho_{\xi}^{(t)}\right|=|\mu|$. This case occurs when $\mu$ has more than one column of length $r_{t-1}$ (i.e., $\mu_{r_{t-1}} \geq 2$ ), and to form $\left(\rho_{\xi}^{(t)} ; \sigma_{\xi}^{(t)}\right)$ we move the corner box in row $r_{t-1}$ down to row $r_{t-1}+1$. (This is either a type-(1) move or, if it happens that $\ell(\nu)=r_{t-1}-1$, a type- $(3)$ move followed by a type-(4) move.) That is, $\sigma_{\xi}^{(t)}=\nu$ and $\rho_{\xi}^{(t)}=\widetilde{\mu}$, where $\widetilde{\mu}$ is the partition $\left(\mu_{1}, \mu_{2}, \ldots, \mu_{r_{t-1}-1}, \mu_{r_{t-1}}-1,1\right)$. In this case the number of rows of $\xi_{t-1}$ containing a + box equals $r_{t-1}+1$, so there is apparently more freedom in the choice of $\xi_{t-1}$ : after labeling every box of $(\widetilde{\mu} ; \nu)$ as + and then inserting - boxes between adjacent + boxes, we still have an additional box to place.

The possibilities are constrained, however, by the analogue of (6.3), which ensures that $\left(\rho_{\xi}^{(t-1)} ; \sigma_{\xi}^{(t-1)}\right) \leq\left(\mu^{\prime} ; \nu\right)$. The forced - boxes in $\xi_{t-1}$ have the shape of the bipartition $\left(\widetilde{\mu}^{\prime} ; \nu\right)$, where $\widetilde{\mu}^{\prime}$ is obtained from $\mu^{\prime}$ by deleting the corner box in row $r_{t-1}$. Hence, $\left(\rho_{\xi}^{(t-1)} ; \sigma_{\xi}^{(t-1)}\right)$ either equals $\left(\mu^{\prime} ; \nu\right)$ or is obtained from $\left(\mu^{\prime} ; \nu\right)$ by moving this same corner box to row $r_{t-1}$ on the $\nu$ side (a type-(3) move) or to row $r_{t-1}+1$ on the $\mu^{\prime}$ side (a type-(1) move, or a type-(3) move followed by a type-(4) move). If $\left(\rho_{\xi}^{(t-1)} ; \sigma_{\xi}^{(t-1)}\right)=\left(\mu^{\prime} ; \nu\right)$, then (6.10) holds. If a move is required, then (6.9) holds. What remains, for this case, is to verify the condition in Lemma 6.6(2) for $j=t-1$.

Suppose that $\ell\left(\rho_{\xi}^{(t-1)}+\sigma_{\xi}^{(t-1)}\right)=r_{t-1}$, which is equivalent to saying that the additional - box in $\xi_{t-1}$ is in row $r_{t-1}$. Then row $r_{t-1}+1$ of $\xi_{t-1}$ consists of a single + box immediately left of the wall; moreover, it is true either that every row of $\xi_{t-1}$ begins with a + or that every row of $\xi_{t-1}$ ends with $\mathrm{a}+$, since the additional - box cannot falsify both statements. So in this event, we are finished.

The other possibility is that $\left(\rho_{\xi}^{(t-1)} ; \sigma_{\xi}^{(t-1)}\right)$ is obtained from $\left(\mu^{\prime} ; \nu\right)$ by moving the corner box in row $r_{t-1}$ on the $\mu^{\prime}$ side to row $r_{t-1}+1$ on the $\mu^{\prime}$ side. Here we observe that since equality holds in (6.6), there cannot be any rearrangement of parts in forming the subordinate bipartition $\left(\rho_{\xi}^{(t-1)} ; \sigma_{\xi}^{(t-1)}\right)$ from $\xi_{t-1}$ (see the comment following (2.8)). So it is not possible that the additional - box in $\xi_{t-1}$ is in a row by itself (immediately right of the wall), 
following row $r_{t-1}+1$, which consists of a single + box (immediately left of the wall). Hence, the additional - box must be in row $r_{t-1}+1$, either before or after the single + box. Again, it follows that either every row of $\xi_{t-1}$ begins with a + or every row of $\xi_{t-1}$ ends with a + . If the additional box comes before the + box in row $r_{t-1}+1$, then that row ends with a box immediately left of the wall. If the additional - box comes after the + box, and therefore right of the wall, then the fact that it is brought to the left of the wall in forming $\left(\rho_{\xi}^{(t-1)} ; \sigma_{\xi}^{(t-1)}\right)$ implies that there is a row of $\xi_{t-1}$ which contains no - boxes right of the wall, and therefore ends with a box immediately left of the wall. So the condition in Lemma 6.6(2) holds.

Case 4: $\ell\left(\rho_{\xi}^{(t)}+\sigma_{\xi}^{(t)}\right)=r_{t-1}+1$ and $\left|\rho_{\xi}^{(t)}\right| \neq|\mu|$. This case occurs when all columns of $\mu$ have length $r_{t-1}, \ell(\nu)=r_{t-1}-1$, and to form $\left(\rho_{\xi}^{(t)} ; \sigma_{\xi}^{(t)}\right)$ from $(\mu ; \nu)$ we move the corner box in row $r_{t-1}-1$ on the $\nu$ side down to row $r_{t-1}+1$ on the $\mu$ side. (This is a type-(3) move followed by two type-(4) moves, or a type-(3) move followed by a single type-(4) move of two boxes.) As in the previous case, after labeling every box of $\left(\rho_{\xi}^{(t)} ; \sigma_{\xi}^{(t)}\right)$ as + and then inserting - boxes between adjacent + boxes, we have to place one additional - box to form $\xi_{t-1}$. Again, the possibilities are constrained by the fact that $\left(\rho_{\xi}^{(t-1)} ; \sigma_{\xi}^{(t-1)}\right) \leq\left(\mu^{\prime} ; \nu\right)$. The forced - boxes in $\xi_{t-1}$ have the shape of the bipartition $\left(\mu^{\prime} ; \widetilde{\nu}\right)$, where $\widetilde{\nu}$ is obtained from $\nu$ by deleting the corner box in row $r_{t-1}-1$. Hence, $\left(\rho_{\xi}^{(t-1)} ; \sigma_{\xi}^{(t-1)}\right)$ either equals $\left(\mu^{\prime} ; \nu\right)$ or is obtained from $\left(\mu^{\prime} ; \nu\right)$ by moving this same corner box to row $r_{t-1}$ on the $\nu$ side or to row $r_{t-1}+1$ on the $\mu^{\prime}$ side. If $\left(\rho_{\xi}^{(t-1)} ; \sigma_{\xi}^{(t-1)}\right)=\left(\mu^{\prime} ; \nu\right)$, then (6.10) holds. If a move is required, then (6.9) holds. The verification that the condition in Lemma 6.6(2) holds for $j=t-1$ is almost identical to the previous case.

Theorem 6.9 completes the proof of Theorem 1.2(3).

\section{$\S 7 . \quad$ Regularity in codimension 1}

In this last section, we prove that every enhanced nilpotent orbit closure is regular in codimension 1. Of course, this would be an immediate consequence of Conjecture 1.1. However, the results in this section hold over any algebraically closed field $\mathbb{F}$, unlike the proposed method of proof of Conjecture 1.1, which requires $\mathbb{F}$ to be $\mathbb{C}$.

We first prove the smoothness of certain unions of orbits in the enhanced nilpotent cone $V \times \mathcal{N}$. As before, $n$ denotes the dimension of $V$. If $\lambda$ is a 
partition of $n$, we write $U_{\lambda}$ for $V \times \mathcal{O}_{\lambda}$, which is a locally closed subvariety of $V \times \mathcal{N}$. Let $\mathcal{Q}_{\lambda}$ be the subset of $\mathcal{Q}_{n}$ consisting of bipartitions $(\mu ; \nu)$ such that $\mu+\nu=\lambda$.

Proposition 7.1. Let $\lambda$ be any partition of $n$.

(1) The variety $U_{\lambda}$ is the union of the orbits $\mathcal{O}_{\mu ; \nu}$ for $(\mu ; \nu) \in \mathcal{Q}_{\lambda}$.

(2) For $(\rho ; \sigma),(\mu ; \nu) \in \mathcal{Q}_{\lambda}, \mathcal{O}_{\rho ; \sigma} \subset \overline{\mathcal{O}_{\mu ; \nu}}$ if and only if $\rho_{i} \leq \mu_{i}$ for all $i$.

(3) For $(\mu ; \nu) \in \mathcal{Q}_{\lambda}$,

$$
\overline{\mathcal{O}_{\mu ; \nu}} \cap U_{\lambda}=\left\{(v, x) \in U_{\lambda} \mid x^{\mu_{i}} v \in \operatorname{im}\left(x^{\lambda_{i}}\right), \text { for all } i\right\} .
$$

(4) In $U_{\lambda}$, the closure of each orbit is smooth; that is, for every $(\mu ; \nu) \in \mathcal{Q}_{\lambda}$, $\overline{\mathcal{O}_{\mu ; \nu}} \cap U_{\lambda}$ is smooth.

Proof. Part (1) is equivalent to (2.2). Part (2) follows from Lemma 2.5. Using the normal basis given in Lemma 2.4, one sees that if $(v, x) \in \mathcal{O}_{\mu ; \nu} \subset$ $U_{\lambda}$, then for all $i$,

$$
\min \left\{s \mid x^{s} v \in \operatorname{im}\left(x^{\lambda_{i}}\right)\right\}=\mu_{i} .
$$

Combining this with part (2), we deduce part (3). From this part (4) follows, because the projection $(v, x) \mapsto x$ exhibits $\overline{\mathcal{O}_{\mu ; \nu}} \cap U_{\lambda}$ as a vector bundle over $\mathcal{O}_{\lambda}$, where the fiber over $x$ is the vector subspace $\bigcap_{i}\left(x^{\mu_{i}}\right)^{-1}\left(\operatorname{im}\left(x^{\lambda_{i}}\right)\right)$ of $V$. (Incidentally, this subspace can alternatively be described as $\sum_{i} x^{\nu_{i}}\left(\operatorname{ker}\left(x^{\lambda_{i}}\right)\right)$.)

If $0 \leq m \leq n$ and $\pi$ is a partition of $n-m$, define

$$
U_{m, \pi}=\left\{(v, x) \in V \times \mathcal{N}|\operatorname{dim} \mathbb{F}[x] v=m, x|_{V / \mathbb{F}[x] v} \in \mathcal{O}_{\pi}\right\}
$$

which is a locally closed subvariety of $V \times \mathcal{N}$. Here $\mathbb{F}[x] v$ is the span of the elements $x^{i} v$ for all $i$, which is obviously an $x$-stable subspace of $V$; since $x$ is nilpotent, to say that $\operatorname{dim} \mathbb{F}[x] v=m$ is to say that $m$ is minimal such that $x^{m} v=0$.

Let $\mathcal{Q}_{m, \pi}$ be the subset of $\mathcal{Q}_{n}$ consisting of bipartitions $(\mu ; \nu)$ such that $\mu_{1}=m$ and $\mu[1]+\nu=\pi$, where $\mu[1]$ denotes the partition $\left(\mu_{2}, \mu_{3}, \ldots\right)$. The map $(\mu ; \nu) \mapsto \mu+\nu$ gives a bijection

$$
\mathcal{Q}_{m, \pi} \longleftrightarrow\left\{\lambda \in \mathcal{P}_{n} \mid \lambda_{1} \geq \pi_{1} \geq \lambda_{2} \geq \pi_{2} \geq \cdots\right\} .
$$

Proposition 7.2. Let $m$ and $\pi$ be as above.

(1) The variety $U_{m, \pi}$ is the union of the orbits $\mathcal{O}_{\mu ; \nu}$ for $(\mu ; \nu) \in \mathcal{Q}_{m, \pi}$. 
(2) For $(\rho ; \sigma),(\mu ; \nu) \in \mathcal{Q}_{m, \pi}, \mathcal{O}_{\rho ; \sigma} \subset \overline{\mathcal{O}_{\mu ; \nu}}$ if and only if $\sigma_{i} \leq \nu_{i}$ for all $i$, which in turn happens if and only if $\mathcal{O}_{\rho+\sigma} \subset \overline{\mathcal{O}_{\mu+\nu}}$.

(3) For $(\mu ; \nu) \in \mathcal{Q}_{m, \pi}$,

$\overline{\mathcal{O}_{\mu ; \nu}} \cap U_{m, \pi}=\left\{(v, x) \in U_{m, \pi} \mid x^{m+\nu_{i}}\left(\left(x^{\pi_{i}}\right)^{-1}(\mathbb{F}[x] v)\right)=0\right.$, for all $\left.i\right\}$.

(4) In $U_{m, \pi}$, the closure of each orbit is smooth; that is, for every $(\mu ; \nu) \in$ $\mathcal{Q}_{m, \pi}, \overline{\mathcal{O}_{\mu ; \nu}} \cap U_{m, \pi}$ is smooth.

Proof. Part (1) is proved in [AH, Lemma 2.5], and part (2) follows from Lemma 2.5. Using the basis defined in [AH, Lemma 2.5], one sees that if $(v, x) \in \mathcal{O}_{\mu ; \nu} \subset U_{m, \pi}$, then for all $i$,

$$
\min \left\{s \mid x^{s}\left(\left(x^{\pi_{i}}\right)^{-1}(\mathbb{F}[x] v)\right)=0\right\}=m+\nu_{i} .
$$

(To verify that the minimum is at least $m+\nu_{i}$, note that the basis element $w_{i, \mu_{i}+\nu_{i}}$ belongs to $\left(x^{\pi_{i}}\right)^{-1}(\mathbb{F}[x] v)$, and that $x^{m+\nu_{i}-1}\left(w_{i, \mu_{i}+\nu_{i}}\right) \neq 0$.) Combining this with part (2), we deduce part (3).

It remains to prove part (4). Let $Z_{m, \pi}$ be the variety of triples $(W, y, z)$ where $W$ is an $m$-dimensional subspace of $V, y$ is a nilpotent endomorphism of $W$ which belongs to $\mathcal{O}_{(m)}$, and $z$ is a nilpotent endomorphism of $V / W$ which belongs to $\mathcal{O}_{\pi}$. It is clear that $Z_{m, \pi}$ is a homogeneous variety for $\mathrm{GL}(V)$. We have a $\mathrm{GL}(V)$-equivariant fiber bundle

$$
\psi: U_{m, \pi} \rightarrow Z_{m, \pi}:(v, x) \mapsto\left(\mathbb{F}[x] v,\left.x\right|_{\mathbb{F}[x] v},\left.x\right|_{V / \mathbb{F}[x] v}\right),
$$

in which the fiber $\psi^{-1}(W, y, z)$ equals $\left(W \backslash \operatorname{ker}\left(y^{m-1}\right)\right) \times A_{W, y, z}$, where

$$
A_{W, y, z}=\left\{x \in \mathfrak{g l}(V)^{W}|x|_{W}=y,\left.x\right|_{V / W}=z\right\},
$$

an affine-linear subspace of $\mathfrak{g l}(V)^{W}$ (the parabolic subalgebra of $\mathfrak{g l}(V)$ stabilizing $W$ ). By parts $(1)$ and $(2), \psi$ restricts to a $\mathrm{GL}(V)$-equivariant fiber bundle

$$
\psi_{\mu ; \nu}: \overline{\mathcal{O}_{\mu ; \nu}} \cap U_{m, \pi} \rightarrow Z_{m, \pi}
$$

in which the fiber $\psi_{\mu ; \nu}^{-1}(W, y, z)$ equals $\left(W \backslash \operatorname{ker}\left(y^{m-1}\right)\right) \times \overline{A_{\mu ; \nu}}$, where

$$
\overline{A_{\mu ; \nu}}=\overline{\mathcal{O}_{\mu+\nu}} \cap A_{W, y, z} .
$$

So it suffices to show that $\overline{A_{\mu ; \nu}}$ is smooth. By part (3),

$$
\overline{A_{\mu ; \nu}}=\left\{x \in A_{W, y, z} \mid x^{m+\nu_{i}}\left(W+\operatorname{ker}\left(z^{\pi_{i}}\right)\right)=0, \text { for all } i\right\},
$$


where $W+\operatorname{ker}\left(z^{\pi_{i}}\right)$ denotes the preimage of $\operatorname{ker}\left(z^{\pi_{i}}\right)$ under the projection $V \rightarrow V / W$. Fixing a base point $x_{0} \in A_{W, y, z}$, one has $A_{W, y, z}=x_{0}+\mathfrak{n}^{W}$, where $\mathfrak{n}^{W}$ is the nilpotent radical of $\mathfrak{g l}(V)^{W}$. For any $k$, the matrix coefficients of $x^{k}-x_{0}^{k}$ are linear functions of $x-x_{0} \in \mathfrak{n}^{W}$, so the condition $x^{m+\nu_{i}}(W+$ $\left.\operatorname{ker}\left(z^{\pi_{i}}\right)\right)=0$ translates into a linear condition on $x-x_{0}$. Hence, $\overline{A_{\mu ; \nu}}$ is an affine-linear subspace of $\mathfrak{g l}(V)^{W}$ and is smooth, as required.

TheOREM 7.3. For every $(\mu ; \nu) \in \mathcal{Q}_{n}, \overline{\mathcal{O}_{\mu ; \nu}}$ is regular in codimension 1.

Proof. Let $\lambda=\mu+\nu$, let $m=\mu_{1}$, and let $\pi=\mu[1]+\nu$. Suppose that $\mathcal{O}_{\rho ; \sigma}$ has codimension 1 in $\overline{\mathcal{O}_{\mu ; \nu}}$. From the description of covering relations given in Section 2.5 and the dimension formula (2.6), it follows that either $(\rho ; \sigma) \in \mathcal{Q}_{\lambda}$ (in the case of a type-(3) move of a single box) or $(\rho ; \sigma) \in \mathcal{Q}_{m, \pi}$ (in the case of a type-(4) move of a single box). So $\mathcal{O}_{\rho ; \sigma}$ is contained in either $\overline{\mathcal{O}_{\mu ; \nu}} \cap U_{\lambda}$ or $\overline{\mathcal{O}_{\mu ; \nu}} \cap U_{m, \pi}$, both of which are open in $\overline{\mathcal{O}_{\mu ; \nu}}$ and smooth by Propositions 7.1 and 7.2. So $\overline{\mathcal{O}_{\mu ; \nu}}$ is smooth at all points of $\mathcal{O}_{\rho ; \sigma}$, proving the result.

Acknowledgments. We are grateful to C. Johnson for sending us an early version of $[\mathrm{J}]$ and to D. Nakano for helpful conversations. We also thank the referee for helpful comments.

\section{REFERENCES}

[AH] P. N. Achar and A. Henderson, Orbit closures in the enhanced nilpotent cone, Adv. Math. 219 (2008), 27-62.

[B] A. Broer, Normal nilpotent varieties in $F_{4}$, J. Algebra 207 (1998), 427-448.

[D] S. Donkin, The normality of closures of conjugacy classes of matrices, Invent. Math. 101 (1990), 717-736.

[FGT] M. Finkelberg, V. Ginzburg, and R. Travkin, Mirabolic affine Grassmannian and character sheaves, Selecta Math. (N.S.) 14 (2009), 607-628.

[J] C. P. Johnson, Enhanced nilpotent representations of a cyclic quiver, preprint, arXiv:1004.3595v1 [math.RT]

[Ka1] S. Kato, An exotic Deligne-Langlands correspondence for symplectic groups, Duke Math. J. 148 (2009), 305-371.

[Ka2] - Deformations of nilpotent cones and Springer correspondences, Amer. J. Math. 133 (2011), 519-553.

[Kr] H. Kraft, Closures of conjugacy classes in $G_{2}$, J. Algebra 126 (1989), 454-465.

[KP1] H. Kraft and C. Procesi, Closures of conjugacy classes of matrices are normal, Invent. Math. 53 (1979), 224-247.

[KP2] - On the geometry of conjugacy classes in classical groups, Comment. Math. Helv. 57 (1982), 539-602.

[M] A. Maffei, Quiver varieties of type A, Comment. Math. Helv. 80 (2005), 1-27.

[S1] E. Sommers, Normality of nilpotent varieties in $E_{6}$, J. Algebra 270 (2003), 288306 . 
[S2] - Normality of very even nilpotent varieties in $D_{2 l}$, Bull. Lond. Math. Soc. 37 (2005), 351-360.

[T] R. Travkin, Mirabolic Robinson-Shensted-Knuth correspondence, Selecta Math. (N.S.) 14 (2009), 727-758.

Pramod N. Achar

Department of Mathematics

Louisiana State University

Baton Rouge, Louisiana 70803

USA

pramod@math.1su.edu

Anthony Henderson

School of Mathematics and Statistics

University of Sydney NSW 2006

Australia

anthony.henderson@sydney . edu. au

Benjamin F. Jones

Department of Mathematics, Statistics, and Computer Science

University of Wisconsin-Stout

Menomonie, Wisconsin 54751

$U S A$

jonesbe@uwstout. edu 